\title{
REPRESENTATIONS AND ISOMORPHISM IDENTITIES FOR INFINITELY DIVISIBLE PROCESSES
}

\author{
BY JAN ROSIŃSKI ${ }^{1}$ \\ University of Tennessee
}

\begin{abstract}
We propose isomorphism-type identities for nonlinear functionals of general infinitely divisible processes. Such identities can be viewed as an analogy of the Cameron-Martin formula for Poissonian infinitely divisible processes but with random translations. The applicability of such tools relies on precise understanding of Lévy measures of infinitely divisible processes and their representations, which are studied here in full generality. We illustrate this approach on examples of squared Bessel processes, Feller diffusions, permanental processes, as well as Lévy processes.
\end{abstract}

1. Introduction. Let $G=\left(G_{t}\right)_{t \in T}$ be a centered Gaussian process over an arbitrary set $T$. The Cameron-Martin formula says that for every random variable $\xi$ in the $L^{2}$-closure of the subspace spanned by $G$ and for any measurable functional $F: \mathbb{R}^{T} \mapsto \mathbb{R}$

$$
\mathbb{E}\left[F\left(\left(G_{t}+\phi(t)\right)_{t \in T}\right)\right]=\mathbb{E}\left[F\left(\left(G_{t}\right)_{t \in T}\right) e^{\xi-\frac{1}{2} \mathbb{E} \xi^{2}}\right],
$$

where $\phi(t)=\mathbb{E}\left(\xi G_{t}\right)$. This formula has many applications, including SDEs and SPDEs driven by Gaussian random fields. It can also be viewed as an isomorphism identity between functionals of a translated Gaussian process and the corresponding functionals of the untranslated process, under the changed probability measure.

It is well known that (1.1) does not extend to the Poissonian case. Indeed, it is easy to show that if $Y=\left(Y_{t}\right)_{t \in[0,1]}$ is a Poisson process, then there is no function $\psi:[0,1] \rightarrow \mathbb{R}, \psi \neq \equiv 0$, such that

$$
\mathbb{E}\left[F\left(\left(Y_{t}+\psi(t)\right)_{t \in[0,1]}\right)\right]=\mathbb{E}\left[F\left(\left(Y_{t}\right)_{t \in[0,1]}\right) \eta\right]
$$

for all measurable functionals $F: \mathbb{R}^{[0,1]} \mapsto \mathbb{R}$ and some random variable $\eta \geq 0$ with $\mathbb{E} \eta=1$.

In this paper, we propose isomorphism identities based on random translations as follows. Let $X=\left(X_{t}\right)_{t \in T}$ be an infinitely divisible process over a general set $T$ (i.e., a process whose finite dimensional distributions are infinitely divisible).

Received July 2016; revised August 2017.

${ }^{1}$ Supported in part by the Simons Foundation Grant 281440.

MSC2010 subject classifications. Primary 60E07, 60G15, 60G17, 60G51; secondary 60G60, 60G99.

Key words and phrases. Infinitely divisible process, Lévy measure on path spaces, isomorphism identities, stochastic integral representations, series representations, Dynkin isomorphism theorem. 
Then, for every process $Z=\left(Z_{t}\right)_{t \in T}$ independent of $X$, whose distribution is absolutely continuous with respect to Lévy measure of $X$, there exists a measurable function $g: \mathbb{R}^{T} \mapsto \mathbb{R}_{+}$such that for any measurable functional $F: \mathbb{R}^{T} \mapsto \mathbb{R}$

$$
\mathbb{E}\left[F\left(\left(X_{t}+Z_{t}\right)_{t \in T}\right)\right]=\mathbb{E}\left[F\left(\left(X_{t}\right)_{t \in T}\right) g(X)\right] .
$$

Relation (1.2) was inspired by Dynkin's isomorphism theorem [5], where $Z=$ $\left(Z_{x}\right)_{x \in E}$ is the total accumulated local time at a state $x$ of a strongly symmetric transient Markov process with the state space $E$ and $X=\left(X_{x}\right)_{x \in E}$ is the squared associated Gaussian process; see Marcus and Rosen [15], Chapter 8. Since the process $X$ is infinitely divisible by results of Eisenbaum [6] and Eisenbaum and Kaspi [8], and $Z$ (over $T=E$ ) satisfies assumptions of (1.2), Dynkin's isomorphism theorem holds as a special case of (1.2). See Example 4.5 for details. Actually, we present this example in a more general setting of permanental processes, following the work of Eisenbaum and Kaspi [9].

There are two basic directions of applying identity (1.2). The first one is to start with a process $Z=\left(Z_{t}\right)_{t \in T}$ of interest, associate with it (possibly) easier to handle the infinitely divisible process $X=\left(X_{t}\right)_{t \in T}$ whose Lévy measure dominates the law of $Z$, and transfer path properties of $X$ to $Z$ via isomorphism (1.2). Using Dynkin's isomorphism theorem, Marcus and Rosen derived many results for local times of Markov processes, including Lévy processes; see, for example, [14], [15]. Another direction of applications of (1.2) is much harder, to derive information about $X$ by utilizing $Z$. One way to approach it is to consider the "converse" version of (1.2) which expresses $X$ as the process $X+Z$ with changed measure. Proposition 4.13 is proven in this spirit. In Sections 4 and 5, we present direct and converse versions of (1.2).

In Section 5, we link isomorphism identity (1.2) with series representations of infinitely divisible processes. This yields more insight into the structure of the admissible translation $Z$, which could be viewed as the zero-term in the series expansion of $X$; see Theorem 5.6. Such representations have proven useful in the study of path regularities of infinitely divisible processes; see, for example, Talagrand [26], Chapter 11.

Successful implementation of isomorphism identities requires precise understanding of Lévy measures of processes, which are defined on path spaces with the usual cylindrical $\sigma$-algebras (as opposed to $\sigma$-rings in [12] and [16]). Section 2 contains systematic development of Lévy measures and spectral representations based on lecture notes [23]. We view Lévy measures as "laws of processes" defined on possibly infinite measure spaces and call such "processes" representations of Lévy measures. Properties of Lévy measures are defined by properties of their representations. Transfer of regularity property (Theorem 3.4) puts the Lévy measure on the same Borel function space where paths of the corresponding infinitely divisible processes belong. This allows to relate path properties of processes and representations of their Lévy measures. 
Throughout this paper, we illustrate general concepts by selected examples of infinitely divisible processes. For this purpose, we have chosen Lévy processes, squared Bessel processes, Feller diffusions, general compound Poisson processes and permanental processes. Some of these processes are presented in their simplest forms in order to not obscure ideas by technical complications. This list of examples can be extended by many processes of interest, including cylindrical Lévy processes in Banach spaces of [1], which can be considered as infinitely divisible processes defined on $T=\mathbb{R} \times E^{\prime}$, where $E$ is a Banach space, self-decomposable fields [2] and multidimensional infinitely divisible processes, which become onedimensional after enlarging the index set.

The paper is organized as follows. Section 2 presents a detailed study of Lévy measures on path spaces. Theorem 2.8 shows existence and uniqueness of such measures for every Poissonian infinitely divisible processes. Theorem 2.12 characterizes the $\sigma$-finiteness of Lévy measures and Theorem 2.14 characterizes Poissonian infinitely divisible process having not $\sigma$-finite Lévy measures. The concept of representations of Lévy measures is introduced and discusses examples in the final part of Section 2. Section 3 gives a Lévy-Itô representation for general infinitely divisible processes (see Theorem 3.2) and Theorem 3.4 states the above mentioned transfer of regularity property. In Section 4, isomorphism identities are given in Theorems 4.1, 4.3 and 4.4. The Dynkin isomorphism theorem for permanental processes is discussed in Example 4.5. Proposition 4.7 characterizes processes satisfying an abstract form of Dynkin's isomorphism, first considered in [7], Lemma 3.1. In the final part of this section, applications of isomorphism identities for Lévy processes are given. Section 5 relates representations of Lévy measures of Section 2 to series representations of Poissonian infinitely divisible processes. As examples, we give series representations of Feller diffusions and squared Bessel processes. Then we connect such representations with isomorphism identities in Theorem 5.6. Section 6 contains the proofs of results from Sections 2-5.

Recall that a stochastic process $X=\left(X_{t}\right)_{t \in T}$, with an arbitrary index set $T$, is said to be Poissonian infinitely divisible if its all finite dimensional marginal distributions are infinitely divisible without a Gaussian part.

Throughout this paper, an identity as (1.2) reads: if one side exists, then the other does and they are equal.

\section{Lévy measures on path spaces.}

2.1. Definitions and preliminaries. Lévy measures of probability laws on $\mathbb{R}^{d}$ are usually defined either on $\mathbb{R}^{d} \backslash\{0\}$ or on $\mathbb{R}^{d}$, in the second case under the assumption that Lévy measures do not charge the origin. Identifying $\mathbb{R}^{d}$ with $\mathbb{R}^{T}$, where $T=\{1, \ldots, d\}$, we have two natural ways to define Lévy measures for infinitely divisible processes over any set $T$. The first way is to define a Lévy measure on the $\sigma$-ring generated by cylindrical subsets of $\mathbb{R}^{T} \backslash\{0\}$, as proposed by Lee [12] and Maruyama [16]. This approach, however, leads to substantial conceptual and 
technical difficulties when $T$ is uncountable. Therefore, we have chosen the second way, to consider Lévy measures on the canonical path space $\left(\mathbb{R}^{T}, \mathscr{B}^{T}\right)$, on which the laws of stochastic processes over $T$ are defined. This approach, in particular, allows us to talk about Lévy measures as "laws of stochastic processes."

Let $\mathbb{R}^{T}$ be the space of all functions $x: T \mapsto \mathbb{R}$, and let $\mathscr{B}^{T}$ denote its cylindrical (product) $\sigma$-algebra. The law of a stochastic process $X=\left(X_{t}\right)_{t \in T}$ is a probability measure $\mu$ on $\left(\mathbb{R}^{T}, \mathscr{B}^{T}\right)$ given by

$$
\mu(A)=\mathbb{P}\left\{\omega:\left(X_{t}(\omega)\right)_{t \in T} \in A\right\}, \quad A \in \mathscr{B}^{T},
$$

and we write $\mathscr{L}(X)=\mu$. For any $S \subset T, X_{S}:=\left(X_{t}\right)_{t \in S}$ is the restriction of the process $X$ to the index set $S$. Similarly, for any $x \in \mathbb{R}^{T}, x_{S}$ denotes the restriction of function $x$ to $S \subset T$. Finally, $0_{S}$ stands for the origin of $\mathbb{R}^{S}$, which will be viewed as a point or the one-point set, depending on the context.

Definition 2.1. A measure $v$ on $\left(\mathbb{R}^{T}, \mathscr{B}^{T}\right)$ is said to be a Lévy measure if the following two conditions hold:

(L1) for every $t \in T \int_{\mathbb{R}^{T}}|x(t)|^{2} \wedge 1 v(d x)<\infty$,

(L2) for every $A \in \mathscr{B}^{T} v(A)=v_{*}\left(A \backslash 0_{T}\right)$, where $v_{*}$ is the inner measure.

The first condition is a technical one, needed for the integral in the LévyKhintchine formula (2.8) to be well defined. The second condition gives the meaning to " $v$ does not charge the origin." If $T$ is countable, then $0_{T} \in \mathscr{B}^{T}$ and (L2) is equivalent to $v\left(0_{T}\right)=0$, which is the usual condition for Lévy measure. If $T$ is uncountable, then $0_{T} \notin \mathscr{B}^{T}$, so that $v\left(0_{T}\right)$ is undefined. However, (L2) still makes sense. We will show that every infinitely divisible process has a unique measure satisfying this definition.

REMARK 2.2. The following condition implies (L2) and is often easier to check: there exists a countable set $T_{0} \subset T$ such that

$$
v\left\{x \in \mathbb{R}^{T}: x_{T_{0}}=0\right\}=0 .
$$

Indeed, from (2.1) we get for any $A \in \mathscr{B}^{T}$

$$
v(A) \geq v_{*}\left(A \backslash 0_{T}\right) \geq v\left(A \backslash\left\{x: x_{T_{0}}=0\right\}\right)=v(A),
$$

which shows (L2).

Throughout this paper, $\hat{T}$ will denote the family of all finite nonempty subsets of the index set $T$,

$$
\hat{T}=\{I \subset T: 0<\operatorname{Card}(I)<\infty\},
$$

so that for any $I \in \hat{T}, \mathbb{R}^{I}$ can be identified with the Euclidean space $\mathbb{R}^{\operatorname{Card}(I)}$. We also set

$$
\hat{T}_{c}:=\{J \subset T: J \text { is nonempty countable }\}
$$


Let $\pi_{S}: \mathbb{R}^{T} \mapsto \mathbb{R}^{S}$ is the projection from $\mathbb{R}^{T}$ onto $\mathbb{R}^{S}, \pi_{S}(x):=x_{S}$. Finally,

$$
\mathscr{B}_{0}^{S}:=\left\{B \in \mathscr{B}^{S}: 0_{S} \notin B\right\} .
$$

The next lemma sheds more light on condition (L2).

LEMMA 2.3. Let $v$ be an arbitrary measure on $\left(\mathbb{R}^{T}, \mathscr{B}^{T}\right)$. Then for every $A \in \mathscr{B}^{T}$

$$
\nu_{*}\left(A \backslash 0_{T}\right)=\sup _{J \in \hat{T}} v\left(A \backslash \pi_{J}^{-1}\left(0_{J}\right)\right) .
$$

Consequently,

$$
\nu^{0}(A):=v_{*}\left(A \backslash 0_{T}\right), \quad A \in \mathscr{B}^{T}
$$

is a measure satisfying (L2) and

$$
v^{0}=v \quad \text { on } \mathscr{B}_{0}^{T} .
$$

Therefore, if $v$ satisfies (L1) then $v^{0}$ is a Lévy measure.

Below we give some equivalent conditions to (L2) that can be easier to verify.

LEMMA 2.4. Let $v$ be a measure on $\left(\mathbb{R}^{T}, \mathscr{B}^{T}\right)$. The following conditions are equivalent to (L2):

(a) for every $T_{0} \in \hat{T}_{c}$ there exists $T_{1} \in \hat{T}_{c}$ such that $T_{0} \subset T_{1}$ and

$$
v\left\{x \in \mathbb{R}^{T}: x_{T_{0}}=0\right\}=v\left\{x \in \mathbb{R}^{T}: x_{T_{0}}=0, x_{T_{1}} \neq 0\right\} .
$$

(b) for every $T_{0} \in \hat{T}_{c}$ with $v\left\{x \in \mathbb{R}^{T}: x_{T_{0}}=0\right\}>0$ there is $t \notin T_{0}$ such that

$$
v\left\{x \in \mathbb{R}^{T}: x_{T_{0}}=0, x(t) \neq 0\right\}>0
$$

(c) either (2.1) is satisfied for some $T_{0} \in \hat{T}_{c}$ or for every $T_{0} \in \hat{T}_{c}$ there is $t \notin T_{0}$ such that (2.4) holds.

REMARK 2.5. Condition (a) was the original condition for a Lévy measure in Rosiński [23]. Condition (b) was communicated to us as equivalent to (a) by Gennady Samorodnitsky. Notice a subtle difference between (b) and the second alternative condition in (c). 
2.2. Lévy-Khintchine and canonical spectral representations. Let $X=$ $\left(X_{t}\right)_{t \in T}$ be an infinitely divisible process, so that for every $I \in \hat{T}$ the random vector $X_{I}$ is infinitely divisible in $\mathbb{R}^{I}$ (which is considered as $\mathbb{R}^{\operatorname{Card}(I)}$ with the inner product $\langle\cdot, \cdot\rangle$ and the norm $|\cdot|)$. By the Lévy-Khintchine representation [24], Theorem 8.1, there exists a unique triplet $\left(\Sigma_{I}, v_{I}, b_{I}\right)$ such that for every $a \in \mathbb{R}^{I}$

$$
\mathbb{E} \exp i\left\langle a, X_{I}\right\rangle
$$

$$
=\exp \left\{-\frac{1}{2}\left\langle a, \Sigma_{I} a\right\rangle+i\left\langle a, b_{I}\right\rangle+\int_{\mathbb{R}^{I}}\left(e^{\langle a, x\rangle}-1-i\langle a, \llbracket x \rrbracket]\right) v_{I}(d x)\right\},
$$

where $\Sigma_{I}$ is a nonnegative definite $I \times I$-matrix, $b_{I} \in \mathbb{R}^{I}$ and $v_{I}$ a Lévy measure, that is, $v_{I}$ is a Borel measure on $\mathbb{R}^{I}$ satisfying

$$
\int_{\mathbb{R}^{I}}\left|x_{I}\right|^{2} \wedge 1 v_{I}(d x)<\infty \quad \text { and } \quad v_{I}\left(0_{I}\right)=0 .
$$

Here, $\mathbb{I} \cdot \rrbracket$ is a fixed truncation function defined as follows. Let $\chi: \mathbb{R} \mapsto \mathbb{R}$ be a bounded measurable function such that $\chi(v)=1+o(|v|)$ as $v \rightarrow 0$ and $\chi(v)=$ $O\left(|v|^{-1}\right)$ as $|v| \rightarrow \infty$. We will call $\chi$ a cutoff function. For example, functions $\mathbf{1}_{\{|v| \leq 1\}},(1 \vee|v|)^{-1},\left(1+|v|^{2}\right)^{-1}$, and the usual cutoff function in a neighborhood of 0 satisfy these conditions. The truncation of $v=\left(v_{1}, \ldots, v_{n}\right) \in \mathbb{R}^{n}$ is defined by

$$
\llbracket v \rrbracket=\left(v_{1} \chi\left(\left|v_{1}\right|\right), \ldots, v_{n} \chi\left(\left|v_{n}\right|\right)\right) .
$$

Similarly, if $y \in \mathbb{R}^{S}$ then $\llbracket y \rrbracket \in \mathbb{R}^{S}$ is given by $\llbracket y \rrbracket(s)=y(s) \chi(y(s)), s \in S$.

From the uniqueness of the triplet $\left(\Sigma_{I}, v_{I}, b_{I}\right)$ in (2.5), the following consistency conditions hold: for every $I, J \in \hat{T}$ with $I \subset J$,

(c1) $\Sigma_{J}$ restricted to $I \times I$ equals $\Sigma_{I}$,

(c2) $b_{J}$ restricted to $I$ equals $b_{I}$,

(c3) $\nu_{J} \circ \pi_{I J}^{-1}=v_{I}$ on $\mathscr{B}_{0}^{I}$,

where $\pi_{I J}: \mathbb{R}^{J} \mapsto \mathbb{R}^{I}$ denotes the natural projection from $\mathbb{R}^{J}$ onto $\mathbb{R}^{I}$. By the Kolmogorov extension theorem, there exist mutually independent centered Gaussian process $G=\left(G_{t}\right)_{t \in T}$ and a Poissonian infinitely divisible process $Y=\left(Y_{t}\right)_{t \in T}$ such that

$$
X \stackrel{d}{=} G+Y,
$$

where for every $I \in \hat{T}, G_{I} \sim N\left(0, \Sigma_{I}\right)$ and

$$
\begin{aligned}
\mathbb{E} \exp i\left\langle a, Y_{I}\right\rangle= & \exp \left\{i\left\langle a, b_{I}\right\rangle+\int_{\mathbb{R}^{I}}\left(e^{\langle a, y\rangle}-1-i\langle a, \llbracket y \rrbracket\rangle\right) \nu_{I}(d y)\right\}, \\
& a \in \mathbb{R}^{I} .
\end{aligned}
$$

The covariance function $\Sigma$ of $G$ restricted to $I \in \hat{T}$, equals $\Sigma_{I}$; similarly, by (c2) there is a path $b: T \mapsto \mathbb{R}$ whose restrictions to $I$ coincide with $b_{I}$. 
DEFINITION 2.6. We say that a family $\left\{v_{I}: I \in \hat{T}\right\}$ of finite dimensional Lévy measures is consistent when it satisfies condition (c3).

It should be noted that a consistent family of finite dimensional Lévy measures is not necessarily a projective system.

EXAmple 2.7. Let $T=\mathbb{N}$ and let $X=\left\{X_{n}\right\}_{n \in \mathbb{N}}$ be an i.i.d. sequence of Poisson random variables with mean 1 . The Lévy measure of $X_{\{1, \ldots, n\}}=\left(X_{1}, \ldots, X_{n}\right)$ is given by

$$
v_{\{1, \ldots, n\}}=\sum_{k=1}^{n} \delta_{0_{\{1, \ldots, k-1\}}} \otimes \delta_{1} \otimes \delta_{0_{\{k+1, \ldots, n\}}} .
$$

Therefore, if $I=\{1, \ldots, n\}$ and $J=\{1, \ldots, r\}$ with $I \subset J$,

$$
v_{J} \circ \pi_{I J}^{-1}=v_{I}+(r-n) \delta_{0_{I}}
$$

which shows that $\left\{v_{I}: I \in \hat{T}\right\}$ is not a projective system of measures.

This fact makes "glueing together" $v_{I}$ 's more complicated than it would be for projective systems. Nevertheless, we have the following.

THEOREM 2.8. Let $Y=\left(Y_{t}\right)_{t \in T}$ be a Poissonian infinitely divisible process as in (2.6). Then there exist a unique Lévy measure $v$ on $\left(\mathbb{R}^{T}, \mathscr{B}^{T}\right)$ and a shift function $b \in \mathbb{R}^{T}$ such that for every $I \in \hat{T}$ and $a \in \mathbb{R}^{I}$

$$
\mathbb{E} \exp i \sum_{t \in I} a_{t} Y_{t}=\exp \left\{\int_{\mathbb{R}^{T}}\left(e^{i\left\langle a, x_{I}\right\rangle}-1-i\left\langle a, \llbracket x_{I} \rrbracket\right|\right) v(d x)+i\left\langle a, b_{I}\right\rangle\right\} .
$$

Therefore, for any consistent system of Lévy measures $\left\{v_{I}: I \in \hat{T}\right\}$ there exists a unique Lévy measure $v$ on $\left(\mathbb{R}^{T}, \mathscr{B}^{T}\right)$ such that

$$
v \circ \pi_{I}^{-1}=v_{I} \quad \text { on } \mathscr{B}_{0}^{I}, I \in \hat{T} .
$$

Furthermore, if $\rho$ is a measure such that $\rho \circ \pi_{I}^{-1}=v_{I}$ on $\mathscr{B}_{0}^{I}$ for all $I \in \hat{T}$, then $v=\rho^{0} \leq \rho .($ cf. Lemma 2.3.)

COROLlary 2.9 (Lévy-Khintchine representation). Let $X=\left(X_{t}\right)_{t \in T}$ be an infinitely divisible process. Then there exists a unique triplet $(\Sigma, v, b)$ consisting of a nonnegative definite function $\Sigma$ on $T \times T$, a Lévy measure $v$ on $\left(\mathbb{R}^{T}, \mathscr{B}^{T}\right)$ and a function $b \in \mathbb{R}^{T}$ such that for every $I \in \hat{T}$ and $a \in \mathbb{R}^{I}$

$\mathbb{E} \exp i \sum_{t \in I} a_{t} X_{t}$

$$
=\exp \left\{-\frac{1}{2}\left\langle a, \Sigma_{I} a\right\rangle+\int_{\mathbb{R}^{T}}\left(e^{i\left\langle a, x_{I}\right\rangle}-1-i\left\langle a, \llbracket x_{I} \rrbracket\right\rangle\right) v(d x)+i\left\langle a, b_{I}\right\rangle\right\},
$$


where $\Sigma_{I}$ is the restriction of $\Sigma$ to $I \times I .(\Sigma, v, b)$ is called the generating triplet of $X$. Conversely, given a generating triplet $(\Sigma, v, b)$ as above, there exists an infinitely divisible process $X=\left(X_{t}\right)_{t \in T}$ satisfying (2.8).

Let $Y=\left(Y_{t}\right)_{t \in T}$ be a Poissonian infinitely divisible process with Lévy measure $v$. Let $N$ be a Poisson random measure on $\left(\mathbb{R}^{T}, \mathscr{B}^{T}\right)$ having intensity measure $v$. The existence of such $N$ follows from Kolmogorov's extension theorem. We will work with a stochastic integral of the form

$$
I_{N}(f)=\int_{\mathbb{R}^{T}} f(x)[N(d x)-\chi(f(x)) v(d x)],
$$

where $\chi$ is a cutoff function defined at the beginning of this section. Since $v$ is not necessarily $\sigma$-finite, we take an extra care in handling this integral.

At the outset, notice that the present development works for a Poisson random measure on any measure space $(S, \mathscr{S}, n)$. For the sake of concreteness, we take here $\left(\mathbb{R}^{T}, \mathscr{B}^{T}, v\right)$ and $\chi(v)=\mathbf{1}_{\{|v| \leq 1\}}$. Let $f=\sum_{j=1}^{n} a_{j} \mathbf{1}_{A_{j}}$, where $a_{j} \in \mathbb{R}$ and $A_{j}$ are disjoint with $v\left(A_{j}\right)<\infty$. We have

$$
I_{N}(f)=\sum_{j \in J_{0}} a_{j}\left[N\left(A_{j}\right)-v\left(A_{j}\right)\right]+\sum_{j \in J_{1}} a_{j} N\left(A_{j}\right)=S_{0}+S_{1},
$$

where $J_{0}=\left\{j \leq n:\left|a_{j}\right| \leq 1\right\}$ and $J_{1}=\left\{j \leq n:\left|a_{j}\right|>1\right\}$. Hence

$$
\begin{aligned}
\mathbb{E}\left(\left|I_{N}(f)\right| \wedge 1\right) & \leq \mathbb{E}\left(\left|S_{0}\right| \wedge 1\right)+\mathbb{E}\left(\left|S_{1}\right| \wedge 1\right) \leq\left(\mathbb{E}\left|S_{0}\right|^{2}\right)^{1 / 2}+\mathbb{E}\left(\left|S_{1}\right| \wedge 1\right) \\
& \leq\left(\sum_{j \in J_{0}} a_{j}^{2} v\left(A_{j}\right)\right)^{1 / 2}+\sum_{j \in J_{1}} \mathbb{E}\left(\left|a_{j} N\left(A_{j}\right)\right| \wedge 1\right) \\
& \leq\left(\int|f|^{2} \wedge 1 d v\right)^{1 / 2}+\sum_{j \in J_{1}} \mathbb{E}\left[\left(\left|a_{j}\right|^{2} \wedge 1\right) N\left(A_{j}\right)\right] \\
& \leq\left(\int|f|^{2} \wedge 1 d v\right)^{1 / 2}+\int|f|^{2} \wedge 1 d \nu
\end{aligned}
$$

Since $\mathbb{E}\left(\left|I_{N}(f)\right| \wedge 1\right) \leq 1$, we infer that

$$
\mathbb{E}\left(\left|I_{N}(f)\right| \wedge 1\right) \leq 2\left(\int|f|^{2} \wedge 1 d v\right)^{1 / 2}
$$

A change of the cut-off function $\chi$ will result in a change of constant 2 to another universal constant. Therefore, the integral in (2.9) is well defined for any measurable $f: \mathbb{R}^{T} \mapsto \mathbb{R}$ such that $\int|f|^{2} \wedge 1 d \nu<\infty$ by the standard approximation procedure. $I_{N}(f)$ is a Poissonian infinitely divisible random variable with the characteristic function

$$
\mathbb{E} \exp \left(i \theta I_{N}(f)\right)=\exp \left(\int_{\mathbb{R}^{T}}\left(e^{i \theta f(x)}-1-i \theta f(x) \chi(f(x))\right) v(d x)\right) .
$$


Proposition 2.10. Let $Y=\left(Y_{t}\right)_{t \in T}$ be a Poissonian infinitely divisible process with Lévy measure $v$ and a shift function $b$. Let $N$ be a Poisson random measure on $\left(\mathbb{R}^{T}, \mathscr{B}^{T}\right)$ having intensity measure $v$. Then the process $\widetilde{Y}=\left(\widetilde{Y}_{t}\right)_{t \in T}$ given by

$$
\tilde{Y}_{t}=\int_{\mathbb{R}^{T}} x(t)[N(d x)-\chi(x(t)) v(d x)]+b(t), \quad t \in T
$$

has the same distribution as $Y . \widetilde{Y}$ will be called a canonical spectral representation of $Y$.

2.3. Sigma-finiteness of Lévy measures. The $\sigma$-finiteness of measures is an important property but not every Lévy measure is $\sigma$-finite. This is shown in the following simple example.

EXAMPLE 2.11. Let $X=\left(X_{t}\right)_{t \in T}$ be un uncountable family of independent Poisson random variables with parameter 1 . Then, for every $I \in \hat{T}, a \in \mathbb{R}^{I}$,

$$
\mathbb{E} \exp i \sum_{t \in I} a_{t} X_{t}=\exp \left[\sum_{t \in I}\left(e^{i a_{t}}-1\right)\right]=\exp \left[\int_{\mathbb{R}^{T}}\left(e^{i\left\langle a, x_{I}\right\rangle}-1\right) v(d x)\right],
$$

where $v$ is the counting measure of a set $E$ given by

$$
E=\left\{e_{s} \in \mathbb{R}^{T}: s \in T, e_{s}(t)=1 \text { if } t=s \text { and } e_{s}(t)=0 \text { otherwise }\right\} .
$$

We have $\int_{\mathbb{R}^{T}}|x(t)|^{2} \wedge 1 v(d x)=\left|e_{t}(t)\right|^{2}=1$ for every $t \in T$. For $T_{0} \in \hat{T}_{c}$ choose $t \notin T_{0}$, and consider $A=\left\{x: x_{T_{0}}=0, x(t) \neq 0\right\}$. Since $A \cap E=\left\{e_{t}\right\}$, we have $v(A)=1>0$, so that $v$ is Lévy measure of $X$ by Lemma 2.4(c). However, $v$ is not $\sigma$-finite as the counting measure of an uncountable set.

The next theorem gives criteria when a Lévy measure is $\sigma$-finite. Notice a subtle difference between (L2) and (ii).

THEOREM 2.12. Let $v$ be a Lévy measure on $\left(\mathbb{R}^{T}, \mathscr{B}^{T}\right)$. The following are equivalent:

(i) $v$ is $\sigma$-finite;

(ii) $v^{*}\left(0_{T}\right)=0$, where $v^{*}$ is the outer measure;

(iii) $v\left\{x \in \mathbb{R}^{T}: x_{T_{0}}=0\right\}=0$ for some $T_{0} \in \hat{T}_{c}$ [i.e., (2.1) holds].

COROLlary 2.13. A Lévy measure is not $\sigma$-finite if and only if for every $T_{0} \in \hat{T}_{c}$ there exists $t \notin T_{0}$ such that $v\left\{x: x_{T_{0}}=0, x(t) \neq 0\right\}>0$.

ProOF. Condition (c) of Lemma 2.4 divides the Lévy measure into two categories: those which satisfy (2.1), which are $\sigma$-finite by Theorem 2.12, and the others which satisfy the condition of this corollary.

We may ask what Poissonian processes do not have $\sigma$-finite Lévy measures. The next theorem characterizes them. 
THEOREM 2.14. Let $Y=\left(Y_{t}\right)_{t \in T}$ be a Poissonian infinitely divisible process with Lévy measure $v$. Then $v$ is not $\sigma$-finite if and only if $T$ is uncountable and there is a version $\tilde{Y}=\left(\widetilde{Y}_{t}\right)_{t \in T}$ of the process $Y$ such that for every $T_{0} \in \hat{T}_{c}$ there exist $t_{1} \notin T_{0}$ and independent random variables $\xi$ and $\eta$ such that:

(a) $\tilde{Y}_{t_{1}}=\xi+\eta$

(b) $\left(\tilde{Y}_{t}, \xi, \eta: t \in T_{0}\right)$ are jointly Poissonian infinitely divisible;

(c) $\eta$ is nondegenerate and independent of $\left(\widetilde{Y}_{t}, \xi: t \in T_{0}\right)$.

REMARK 2.15. Intuitively, a Poissonian infinitely divisible process has a $\sigma$ finite Lévy measure if and only if there exists $T_{0} \in \hat{T}_{c}$ such that outside of the index set $T_{0}$ the process has no nontrivial independent components.

Proposition 2.16. Let $Y=\left(Y_{t}\right)_{t \in T}$ be a Poissonian infinitely divisible process such that there exists a countable $T_{0} \subset T$ such that every $Y_{t}$ is measurable with respect to $\bar{\sigma}\left(Y_{T_{0}}\right) \mathbb{P}$, the $\mathbb{P}$-completion of $\sigma\left(Y_{t}: t \in T_{0}\right)$. Then $Y$ has a $\sigma$-finite Lévy measure.

PROOF. From the assumption, for every $t \in T$ there exists a Borel measurable function $\Phi_{t}: \mathbb{R}^{T_{0}} \mapsto \mathbb{R}$ such that $Y_{t}=\Phi_{t}\left(Y_{T_{0}}\right)$ a.s. Suppose to the contrary that $v$ is not $\sigma$-finite, so by Theorem 2.14 there exist a version $\tilde{Y}$ and $t_{1} \notin T_{0}$ such that (a)-(c) hold. We also have $\tilde{Y}_{t_{1}}=\Phi_{t_{1}}\left(\tilde{Y}_{T_{0}}\right)$ a.s. Hence, for any $u \in \mathbb{R}$

$$
e^{i u(\xi+\eta)}=\mathbb{E}\left[e^{i u(\xi+\eta)} \mid \tilde{Y}_{T_{0}}, \xi\right]=e^{i u \xi} \mathbb{E}\left[e^{i u \eta} \mid \tilde{Y}_{T_{0}}, \xi\right]=e^{i u \xi} \mathbb{E}\left[e^{i u \eta}\right],
$$

which gives $\left|\mathbb{E}\left[e^{i u \eta}\right]\right|=1$, so that $\eta$ is deterministic. A contradiction.

DEFINITION 2.17. A stochastic process $Y=\left(Y_{t}\right)_{t \in T}$ is said to be separable in probability if there exists $T_{0} \in \hat{T}_{c}$ such that for any $t \in T$ there is a sequence $\left\{s_{n}\right\} \subset T_{0}$ such that $Y_{s_{n}} \stackrel{P}{\rightarrow} Y_{t}$.

From Proposition 2.16, we get the following.

COROllary 2.18. A separable in probability Poissonian infinitely divisible process $Y$ has a $\sigma$-finite Lévy measure.

The fact stated in Corollary 2.18 was also proved by Kabluchko and Stoev [10] by different methods.

REMARK 2.19. Comparing Theorem 2.12(iii) and Corollary 2.18, it seem that the $\sigma$-finiteness of a Lévy measure and the separability in probability of the corresponding Poissonian process are close. However, the separability in probability is a stronger condition. Indeed, let $V=\left\{V_{t}\right\}_{t \in[0,1]}$ be a family of i.i.d. 
Rademacher random variables, $\mathbb{P}\left(V_{t}= \pm 1\right)=1 / 2$, and let $v$ be the probability distribution of $V$ in $\left(\mathbb{R}^{[0,1]}, \mathscr{B}^{[0,1]}\right)$. $v$ trivially satisfies $(2.1)$, so it is a Lévy measure. Let $Y=\left\{Y_{t}\right\}_{t \in[0,1]}$ be the corresponding compound Poisson process; see Example 2.26. Since $\mathbb{P}\left(\left|Y_{t}-Y_{s}\right|>1\right)>1 /(2 e)$ for any $s \neq t, Y$ has finite Lévy measure but is not separable in probability.

2.4. Representations and examples of Lévy measures of processes. A natural way to describe Lévy measures on path spaces is to view them as "laws of processes" defined on possibly infinite measure spaces. Below we formalize this approach.

Definition 2.20. Let $\left\{v_{I}: I \in \hat{T}\right\}$ be a consistent family of finite dimensional Lévy measures, which extends uniquely to a Lévy measure $v$ by Theorem 2.8. A collection of measurable functions $V=\left(V_{t}\right)_{t \in T}$ defined on a measure space $(S, \mathscr{S}, n)$ is said to be a representation of $v$ if for every $I \in \hat{T}$ :

$$
n\left(\left\{s: V_{I}(s) \in B\right\}\right)=v_{I}(B) \quad \text { for every } B \in \mathscr{B}_{0}^{I} .
$$

A representation $V$ is called exact if $n \circ V^{-1}=v$ or, equivalently, if $n \circ V^{-1}$ satisfies (L2). Here, $V$ is viewed as a function from $S$ into $\mathbb{R}^{T}$ given by $V(s)(\cdot)=$ $V_{(\cdot)}(\mathrm{s})$.

Representations of Lévy measures are useful for stochastic integral and series representations of the corresponding infinitely divisible processes while exact representations give precise forms of Lévy measures. The difference between these two representations is a technical one, as it is shown below.

LEMMA 2.21. Any representation of a Lévy measure, defined on a $\sigma$-finite measure space, can be modified to an exact representation by restricting it to a smaller domain.

REMARK 2.22. Any Lévy measure $v$ has an exact representation. Indeed, the evaluation process $V_{t}(x)=x(t), x \in \mathbb{R}^{T}, t \in T$ is an exact representation of $v$ on $(S, \mathscr{S}, n)=\left(\mathbb{R}^{T}, \mathscr{B}^{T}, v\right)$. However, such representation does not give much of information about the Lévy measure because it is too general. Therefore, we are seeking more specific representations on richer structures, such as standard Borel spaces (Borel subsets of Polish spaces; see [11], Chapter 1).

EXAMPLE 2.23 (Lévy processes). Let $Y=\left(Y_{t}\right)_{t \geq 0}$ be a Poissonian-Lévy process determined by $\mathbb{E} e^{i u Y_{t}}=e^{t K(u)}$, where $K$ is the cumulant function given by

$$
K(u)=\int_{\mathbb{R}}\left(e^{i u x}-1-i u \llbracket x \rrbracket\right) \rho(d x)+i u c .
$$


For every $I=\left\{t_{1}, \ldots, t_{n}\right\}$, with $0 \leq t_{1}<\cdots<t_{n}$, and $a=\left(a_{1}, \ldots, a_{n}\right) \in \mathbb{R}^{I}(\equiv$ $\mathbb{R}^{n}$ ) we have

$$
\mathbb{E} \exp i \sum_{k=1}^{n} a_{k} Y_{t_{k}}=\exp \left\{\sum_{k=1}^{n} K\left(u_{k}\right) \Delta t_{k}\right\}
$$

where $\Delta t_{k}=t_{k}-t_{k-1}, u_{k}=\sum_{j=k}^{n} a_{j}$, and $t_{0}=0$. Therefore, the Lévy measure $v_{I}$ of $X_{I}$ is given by

$$
v_{I}(B)=\sum_{k=1}^{n} \int_{\mathbb{R}} \mathbf{1}_{B}\left(v x_{k}\right) \rho(d v) \Delta t_{k}, \quad B \in \mathscr{B}^{n},
$$

where $x_{k} \in \mathbb{R}^{n}, x_{k}=(\underbrace{0, \ldots, 0}_{k-1 \text { times }}, 1, \ldots, 1), k=1, \ldots, n$. Define $V=\left(V_{t}\right)_{t \in T}$ on the half-plane $\mathbb{R}_{+} \times \mathbb{R}$ equipped with a measure $\lambda \otimes \rho$ given by

$$
V_{t}(r, v)=\mathbf{1}_{\{t \geq r\}} v, \quad(r, v) \in \mathbb{R}_{+} \times \mathbb{R},
$$

where $\lambda$ denotes the Lebesgue measure. We first verify that $V$ is a representation of the Lévy measure $v$ of $Y$. Let $I$ be a finite set of indices as above. For any $B \in \mathscr{B}_{0}^{I}$, we have

$$
\begin{aligned}
(\lambda \otimes \rho)\left\{V_{I} \in B\right\} & =\int_{\mathbb{R}} \int_{0}^{\infty} \mathbf{1}_{B}\left(V_{I}(r, v)\right) d r \rho(d v) \\
& =\int_{\mathbb{R}} \sum_{k=1}^{n} \int_{t_{k-1}}^{t_{k}} \mathbf{1}_{B}\left(\mathbf{1}_{\left\{t_{1} \geq r\right\}} v, \ldots, \mathbf{1}_{\{t \geq r\}} v\right) d r \rho(d v) \\
& =\int_{\mathbb{R}} \sum_{k=1}^{n} \mathbf{1}_{B}(\underbrace{0, \ldots, 0}_{k-1 \text { times }}, v, \ldots, v) \rho(d v) \Delta t_{k}=v_{I}(B),
\end{aligned}
$$

as in (2.12). To check that $V$ is exact, it is enough to verify (2.1). Indeed, for $T_{0}=\mathbb{N}$ we have

$$
(\lambda \otimes \rho)\left\{(r, v): \mathbf{1}_{\{n \geq r\}} v=0 \forall n \in \mathbb{N}\right\}=0 .
$$

Thus $V$ in (2.13) is an exact representation of $v$.

EXAmple 2.24 (Squared Bessel processes). Let $Y=\left(Y_{t}\right)_{t \geq 0}$ denote a squared Bessel process of dimension $\beta>0$ starting from 0 . If $\beta \in \mathbb{N}$, then $Y_{t}:=\left\|B_{t}\right\|^{2}$, where $B$ is a $\beta$-dimensional standard Brownian motion. In general, $Y$ is defined as the unique solution of the stochastic differential equation

$$
d Y_{t}=2 \sqrt{Y_{t}} d W_{t}+\beta d t, \quad Y_{0}=0,
$$

where $W$ is a one-dimensional standard Brownian motion. Shiga and Watanabe [25] showed that squared Bessel processes are infinitely divisible and Pitman and 
Yor [17] described their Lévy measures on $C\left(\mathbb{R}_{+}\right)$. We will adapt that characterization to our setting.

Let

$$
U_{+}:=\left\{u \in C\left(\mathbb{R}_{+}\right): u(0)=0, u_{\mid\left(0, t_{0}\right)}>0, u_{\mid\left[t_{0}, \infty\right)}=0 \text { for some } t_{0}>0\right\} .
$$

$U_{+}$is a Borel subset of $C\left(\mathbb{R}_{+}\right)$, on which we consider the Itô measure $n_{+}$of the Brownian positive excursions; see Revuz and Yor [19], Chapter XII. Let $L_{\infty}^{a}(u)$ denote the total accumulated local time of an excursion $u \in U_{+}$at $a>0$. Symbolically,

$$
L_{\infty}^{a}(u)=\int_{0}^{\infty} \delta_{a}(u(t)) d t .
$$

Set $L_{\infty}^{a}(u)=0$ when $a \leq 0$. Define $V=\left(V_{t}\right)_{t \geq 0}$ on a Borel space $\mathbb{R}_{+} \times U_{+}$with a measure $\beta \lambda \otimes n_{+}$by

$$
V_{t}(r, u)=L_{\infty}^{t-r}(u), \quad r \geq 0, u \in U_{+} .
$$

To check that $V$ is an exact representation of the Lévy measure $v$ of $Y$, we invoke an equation given after Theorem 3.2 in Mansuy and Yor [13] which states that

$$
\int_{U_{+}} v(d u) F(u)=\beta \int_{U_{+}} M(d u) \int_{0}^{\infty} d r F\left(u\left((\cdot-r)^{+}\right)\right)
$$

for any measurable functional $F: U_{+} \mapsto \mathbb{R}_{+}$, where $M=n_{+} \circ L_{\infty}^{(\cdot)-1}$. This equation says that $\left(\beta \lambda \otimes n_{+}\right) \circ V^{-1}=v$.

EXAMPLE 2.25 (Feller diffusion). We consider a Feller diffusion $Z=\left(Z_{t}\right)_{t \geq 0}$ without the drift term, which satisfies the stochastic differential equation

$$
d Z_{t}=\sigma \sqrt{Z_{t}} d W_{t}, \quad Z_{0}=a>0,
$$

where $W$ is a one-dimensional standard Brownian motion. By change of time, $Y_{t}=Z_{4 \sigma^{-2} t}$ we have

$$
d Y_{t}=2 \sqrt{Y_{t}} d W_{t}^{\prime}, \quad Y_{0}=a>0,
$$

where $W^{\prime}$ is another standard Brownian motion. Therefore, $Y$ is a 0 -dimensional squared Bessel process whose Lévy measure $v_{0}$ given in the above cited [13], Theorem 3.2. Namely, in the notation of Example 2.24, $v_{0}=a n_{+} \circ L_{\infty}^{(\cdot)}{ }^{-1}$. Therefore, $V=\left(V_{t}\right)_{t \geq 0}$ given by

$$
V_{t}(u)=L_{\infty}^{4^{-1} \sigma^{2} t}(u), \quad u \in U_{+}
$$

is a representation of the Lévy measure of $Z=\left(Z_{t}\right)_{t \geq 0}$ on $\left(U_{+}, \mathscr{B}\left(U_{+}\right), a n_{+}\right)$.

The next example provides a simple illustration for the method of Lemma 2.21. 
EXAMPLE 2.26 (General compound Poisson processes). Let $V=\left\{V_{t}\right\}_{t \in T}$ be a stochastic process and let $\zeta$ a Poisson random variable with mean $\theta$. Let $\left\{V^{(n)}\right\}_{n \in \mathbb{N}}$ be a sequence of independent copies of $V$ and independent of $\zeta$. Then

$$
Y_{t}=\sum_{n=1}^{\zeta} V_{t}^{(n)}, \quad t \in T
$$

is a Poissonian infinitely divisible process such that for every $I \in \hat{T}, a \in \mathbb{R}^{I}$,

$$
\mathbb{E} \exp i \sum_{t \in I} a_{t} Y_{t}=\exp \left\{\theta \mathbb{E}\left(e^{i \sum_{t \in I} a_{t} V_{t}}-1\right)\right\}=\exp \left\{\int_{\mathbb{R}^{I}}\left(e^{i\langle a, y\rangle}-1\right) v_{I}(d y)\right\} .
$$

Thus $V=\left\{V_{t}\right\}_{t \in T}$ is a representation the Lévy measure $v$ of $Y$ on $(\Omega, \mathscr{F}, \theta \mathbb{P})$. By the proof of Lemma 2.21, the restriction $V_{0}$ of $V$ to $\Omega_{0}:=\left\{\omega: V_{T_{0}}(\omega) \neq 0\right\}$ is an exact representation of $\nu$, where $T_{0} \in \hat{T}_{c}$ is such that

$$
\mathbb{P}\left(V_{T_{0}}=0\right)=\inf _{J \in \hat{T}} \mathbb{P}\left\{\omega: V_{J}(\omega)=0\right\} .
$$

3. Lévy-Itô representations and transfer of regularity for Lévy measures. The following proposition is a direct extension of Proposition 2.10 with a similar proof. Thus its proof will be omitted.

Proposition 3.1. Let $X=\left(X_{t}\right)_{t \in T}$ be an infinitely divisible process with the generating triplet $(\Sigma, v, b)$. Suppose $V=\left(V_{t}\right)_{t \in T}$ is a representation of $v$ on $(S, \mathscr{S}, n)$. Let $G=\left(G_{t}\right)_{t \in T}$ be a centered Gaussian process with covariance $\Sigma$ and let $N$ be a Poisson random measure on $(S, \mathscr{S})$ with intensity $n$ such that $G$ and $N$ are independent. Then the process $X^{\prime}=\left(X_{t}^{\prime}\right)_{t \in T}$ given by

$$
X_{t}^{\prime}:=G_{t}+\int_{S} V_{t}(s)\left(N(d s)-\chi\left(V_{t}(s)\right) n(d s)\right)+b(t), \quad t \in T,
$$

is a version of the process $X$.

The next theorem shows that, under some regularity assumptions, spectral representations hold almost surely. Recall Definition 2.17 (separability in probability).

THEOREM 3.2 (Generalized Lévy-Itô representation). Let $X=\left(X_{t}\right)_{t \in T}$ be a separable in probability infinitely divisible process with a separant $T_{0}$ and the generating triplet $(\Sigma, v, b)$. Assume that the probability space is rich enough to support independent of $X$ standard uniform random variable. Then, given a representation $V=\left(V_{t}\right)_{t \in T}$ of $v$ defined on a $\sigma$-finite measure space $(S, \mathscr{S}, n)$, where $\mathscr{S}$ is countably generated (modulo $n$ ), there exist a centered Gaussian process $G=\left(G_{t}\right)_{t \in T}$ with covariance $\Sigma$ and independent of $G$ Poisson random measure $N$ on $(S, \mathscr{S})$ with intensity measure $n$, such that for every $t \in T$ :

$$
X_{t}=G_{t}+\int_{S} V_{t}(s)\left(N(d s)-\chi\left(V_{t}(s)\right) n(d s)\right)+b(t) \quad \text { a.s. }
$$


We illustrate this representation on four examples of infinitely divisible processes.

Corollary 3.3. (a) Lévy processes. Let $Y=\left(Y_{t}\right)_{t \geq 0}$ be a Poissonian-Lévy process as in Example 2.23, with $\chi=\mathbf{1}\{|v| \leq 1\}$. Formula (3.2) applied to $V$ in (2.13) yields the usual Lévy-Itô representation: with probability 1 for all $t \geq 0$,

$$
\begin{aligned}
Y_{t} & =\int_{\mathbb{R}_{+}} \int_{\mathbb{R}} \mathbf{1}_{\{t \geq r\}} v\left(N(d r, d v)-\chi\left(\mathbf{1}_{\{t \geq r\}} v\right) d r \rho(d v)\right)+c t \\
& =\int_{0}^{t} \int_{|v| \leq 1} v(N(d r, d v)-d r \rho(d v))+\int_{0}^{t} \int_{|v|>1} v N(d r, d v)+c t,
\end{aligned}
$$

where $N$ is s Poisson random measure on $\mathbb{R}_{+} \times \mathbb{R}$ with intensity $\lambda \otimes \rho$.

(b) Squared Bessel processes. Let $Y=\left(Y_{t}\right)_{t \geq 0}$ be a squared Bessel process of dimension $\beta>0$ starting from 0 , as in Example 2.24. Formula (3.2) applied to $V$ in (2.14) yields: with probability 1 for all $t \geq 0$,

$$
Y_{t}=\int_{0}^{t} \int_{U_{+}} L_{\infty}^{t-r}(u) N(d r, d u),
$$

where $N$ is s Poisson random measure on $\mathbb{R}_{+} \times U_{+}$with intensity $\beta \lambda \otimes n_{+}$. Therefore, a squared Bessel process $Y$ is a mixed stochastic convolution.

(c) Feller diffusion. Let $Z=\left(Z_{t}\right)_{t \geq 0}$ be a Feller diffusion starting from $a>0$, as in Example 2.25. Formula (3.2) applied to $V$ in (2.15) yields: with probability 1 for all $t \geq 0$,

$$
Z_{t}=\int_{U_{+}} L_{\infty}^{\kappa t}(u) N(d u)
$$

where $\kappa=\sigma^{2} / 4$ and $N$ is $s$ Poisson random measure on $U_{+}$with intensity an $n_{+}$.

(d) Compound Poisson process. Let $Y=\left(Y_{t}\right)_{t \in T}$ be a compound Poisson process generated by a stochastic process $V=\left(V_{t}\right)_{t \in T}$ and $\theta>0$, as in Example 2.26. Suppose that $V$ is separable in probability. Then (3.2) applied to $V$ yields: with probability 1 for all $t \in T$

$$
Y_{t}=\int_{\Omega} V_{t}(\omega) N(d \omega)
$$

where $N$ is s Poisson random measure on $\Omega$ with intensity $\theta \mathbb{P}$.

Next, we consider the transfer of regularity for Lévy measures. In short, this property says that path regularities of infinitely divisible processes are inherited by representations of their Lévy measures. A precise statement follows.

THEOREM 3.4 (Transfer of regularity). Let $X=\left(X_{t}\right)_{t \in T}$ be an infinitely divisible process with a $\sigma$-finite Lévy measure $v$. Assume that paths of $X$ lie in a set $U$ that is a standard Borel space for the $\sigma$-algebra $\mathscr{U}=\mathscr{B}^{T} \cap U$ and also that $U$ 
is an algebraic subgroup of $\mathbb{R}^{T}$ under addition. Then $v$ is concentrated on $U$ in the sense that $v_{*}\left(\mathbb{R}^{T} \backslash U\right)=0$. Therefore, $v$ is well defined on $\mathscr{U}$ and the evaluation process on $(U, \mathscr{U}, v)$ is an exact representation of $v$ and has paths in $U$.

Moreover, any representation $V=\left(V_{t}\right)_{t \in T}$ of $\nu$, given on a $\sigma$-finite measure space $(S, \mathscr{S}, n)$, can be modified to an exact representation $\widetilde{V}=\left(\widetilde{V}_{t}\right)_{t \in T}$ of $v$ with paths in $U$ and such that $n\left\{s: \widetilde{V}_{t}(s) \neq V_{t}(s)\right\}=0$ for every $t \in T$.

Let $T=[0,1]$, for concreteness. Examples of $U$ satisfying the above theorem include such "obvious" spaces as $C[0,1]$ and $D[0,1]$ (the latter one under the Skorohod topology), but they also include nonseparable Banach spaces such as the space of Lipschitz-continuous functions $C^{0,1}[0,1]$ and the space of càdlàg functions of finite variation $B V_{1}[0,1]$ (which are Borel subsets of $C[0,1]$ and $D[0,1]$, resp.).

As an application, consider a càdàg Poissonian infinitely divisible process $Y=$ $\left(Y_{t}\right)_{t \in[0,1]}$. Since $Y$ is right continuous, it is separable in probability, so its Lévy measure $v$ is $\sigma$-finite. By Theorem 3.4, $v$ is concentrated on $D[0,1]$. Using Basse and Rosiński [3], Lemma 3.5, we get $v\left\{\|x\|_{\infty}>r\right\}<\infty$ for any $r>0$. Therefore, the Lévy-Khintchine representation of Theorem 2.8 can be refined to

$$
\begin{aligned}
\mathbb{E} \exp i & \sum_{j=1}^{n} a_{j} Y_{t_{j}} \\
= & \exp \left\{\int_{D[0,1]}\left(e^{i \sum_{j=1}^{n} a_{j} x\left(t_{j}\right)}-1-i \sum_{j=1}^{n} a_{j} x\left(t_{j}\right) \mathbf{1}_{[0,1]}\left(\|x\|_{\infty}\right)\right) v(d x)\right. \\
& \left.+i \sum_{j=1}^{n} a_{j} b\left(t_{j}\right)\right\},
\end{aligned}
$$

where $b \in D[0,1]$ and $\|\cdot\|_{\infty}$ is the supremum norm.

4. Isomorphism identities and spectral representations. We begin with some identities for expectations of functionals of stochastic processes. We present them under different degrees of generality of the assumptions, which is more suitable for applications. For any two $\sigma$-finite measures $\mu$ and $\nu$, we write $\mu \ll v$ when $\mu$ is absolutely continuous with respect to $\nu$, and $\mu \sim \nu$ when these measures are equivalent. We will also write $\mathbb{E}[\xi ; \eta]$ for $\mathbb{E}[\xi \eta]$ and $\mathbb{E}[\xi ; A]:=\mathbb{E}\left[\xi \mathbf{1}_{A}\right]$, and use the convention $0 \cdot \infty=0$.

THEOREM 4.1. Let $X=\left(X_{t}\right)_{t \in T}$ be an infinitely divisible process having a $\sigma$-finite Lévy measure $v$. Let $Z=\left(Z_{t}\right)_{t \in T}$ be a process independent of $X$ such that $\mathscr{L}(Z) \ll v$. Then $\mathscr{L}(X+Z) \ll \mathscr{L}(X)$. Hence, there exists a measurable functional $g: \mathbb{R}^{T} \mapsto \mathbb{R}_{+}$such that for any measurable functional $F: \mathbb{R}^{T} \mapsto \mathbb{R}$

$$
\mathbb{E} F\left(\left(X_{t}+Z_{t}\right)_{t \in T}\right)=\mathbb{E}\left[F\left(\left(X_{t}\right)_{t \in T}\right) ; g(X)\right] .
$$


Theorem 4.1 is a consequence of Theorem 4.3, which itself is deduced from Theorem 4.4 .

REMARK 4.2. (a) From (4.1), it follows that the linear spaces of functionals $F(X+Z)$ of the process $X+Z$ and $F(X)$ of $X$ are isometric under various norms, such as the $L^{p}$-norms, but with respect to possibly different probability measures. This may explain the name "isomorphism theorems" or "isomorphism identities" for results of this kind of formulas.

(b) The processes $Z$ can be viewed as a random translation (or perturbation) of $X$, so that Theorem 4.1 gives a sufficient condition when such translation (perturbation) is "admissible."

The function $g$ in (4.1) has a closed form only in certain cases. Therefore, we will give below another, easier to handle term in place of $g(X)$. We can also impose a slightly weaker condition on the process $Z$ in part (b), than the one in the previous theorem.

THEOREM 4.3. Let $X=\left(X_{t}\right)_{t \in T}$ be an infinitely divisible process of the form $X=G+Y$, where $G=\left(G_{t}\right)_{t \in T}$ is a centered Gaussian process independent of a Poissonian process $Y=\left(Y_{t}\right)_{t \in T}$ having a $\sigma$-finite Lévy measure $v$ and given by its canonical spectral representation

$$
Y_{t}=\int_{\mathbb{R}^{T}} x(t)[N(d x)-\chi(x(t)) v(d x)]+b(t), \quad t \in T .
$$

Here, $N$ is a Poisson random measure with intensity v (see Proposition 2.10). Let $Z=\left(Z_{t}\right)_{t \in T}$ be an arbitrary process independent of $N$.

(a) Suppose that $\mathscr{L}(Z) \ll v$ and let $q:=\frac{d \mathscr{L}(Z)}{d v}$ be the Radon-Nikodym derivative of $\mathscr{L}(Z)$ with respect to $v$. Then for any measurable functional $F: \mathbb{R}^{T} \mapsto \mathbb{R}$

$$
\mathbb{E} F\left(\left(X_{t}+Z_{t}\right)_{t \in T}\right)=\mathbb{E}\left[F\left(\left(X_{t}\right)_{t \in T}\right) ; N(q)\right]
$$

where

$$
N(q)=\int_{\mathbb{R}^{T}} q(x) N(d x) .
$$

Conversely, for any $F$ as above,

$$
\mathbb{E}\left[F\left(\left(X_{t}\right)_{t \in T}\right) ; N(q)>0\right]=\mathbb{E}\left[F\left(\left(X_{t}+Z_{t}\right)_{t \in T}\right)(N(q)+q(Z))^{-1}\right],
$$

where $q(Z)=q\left(\left(Z_{t}\right)_{t \in T}\right)$. Moreover, if $v\{x: q(x)>0\}=\infty$, then $\mathscr{L}(Y+Z)$ and $\mathscr{L}(Y)$ are equivalent.

(b) Suppose that $\mathscr{L}(Z) \ll v+\delta_{0_{T}}$ and let $q:=\frac{d \mathscr{L}(Z)}{d\left(v+\delta_{\left.0_{T}\right)}\right)}$. Then for any measurable functional $F: \mathbb{R}^{T} \mapsto \mathbb{R}$,

$$
\mathbb{E} F\left(\left(X_{t}+Z_{t}\right)_{t \in T}\right)=\mathbb{E}\left[F\left(\left(X_{t}\right)_{t \in T}\right) ; N(q)+q\left(0_{T}\right)\right] .
$$


Conversely, for any $F$ as above,

$$
\begin{aligned}
& \mathbb{E}\left[F\left(\left(X_{t}\right)_{t \in T}\right) ; N(q)+q\left(0_{T}\right)>0\right] \\
& \quad=\mathbb{E}\left[F\left(\left(X_{t}+Z_{t}\right)_{t \in T}\right)\left(N(q)+q(Z)+q\left(0_{T}\right) \mathbf{1}_{U^{c}}(Z)\right)^{-1}\right],
\end{aligned}
$$

where $q(Z)=q\left(\left(Z_{t}\right)_{t \in T}\right)$ and $U \in \mathscr{B}^{T}$ is such that $0_{T} \in U \in \mathscr{B}^{T}$ and $v(U)=0$. Furthermore, $\mathscr{L}(Y+Z)$ and $\mathscr{L}(Y)$ are equivalent if $q\left(0_{T}\right)>0$ or $v\{x: q(x)>$ $0\}=\infty$.

The previous two theorems will be proved as special cases of the next result.

THEOREM 4.4. Let $X=\left(X_{t}\right)_{t \in T}$ be an infinitely divisible process given by

$$
X_{t}=G_{t}+\int_{S} V_{t}(s)\left[N(d s)-\chi\left(V_{t}(s)\right) n(d s)\right]+b(t)
$$

where $V=\left(V_{t}\right)_{t \in T}$ is a representation of the Lévy measure of $X$ defined on a $\sigma$ finite measure space $(S, \mathscr{S}, n), N$ is a Poisson random measure on $(S, \mathscr{S})$ with intensity $n, G=\left(G_{t}\right)_{t \in T}$ is a centered Gaussian process independent of $N$ and $b$ is a shift function. Choose an arbitrary measurable function $q: S \mapsto \mathbb{R}_{+}$such that $\int_{S} q(s) n(d s)=1$. Then for any measurable functional $F: \mathbb{R}^{T} \mapsto \mathbb{R}$

$$
\int_{S} \mathbb{E} F\left(\left(X_{t}+V_{t}(s)\right)_{t \in T}\right) q(s) n(d s)=\mathbb{E}\left[F\left(\left(X_{t}\right)_{t \in T}\right) ; N(q)\right],
$$

where

$$
N(q)=\int_{S} q(s) N(d s) .
$$

Conversely, for any $F$ as above,

$$
\begin{aligned}
& \mathbb{E}\left[F\left(\left(X_{t}\right)_{t \in T}\right) ; N(q)>0\right] \\
& \quad=\int_{S} \mathbb{E}\left[F\left(\left(X_{t}+V_{t}(s)\right)_{t \in T}\right) ;(N(q)+q(s))^{-1}\right] q(s) n(d s) .
\end{aligned}
$$

If $n\{s \in S: q(s)>0\}=\infty$, then

$$
\mathbb{E}\left[F\left(\left(X_{t}\right)_{t \in T}\right)\right]=\int_{S} \mathbb{E}\left[F\left(\left(X_{t}+V_{t}(s)\right)_{t \in T}\right) ;(N(q)+q(s))^{-1}\right] q(s) n(d s) .
$$

Now we will discuss how Dynkin's isomorphism fits into the pattern of Theorems 4.1 and 4.3.

EXAMPLE 4.5 (Dynkin isomorphism for permanental processes).

A positive real-valued stochastic process $Y=\left(Y_{x}\right)_{x \in E}$ over a set $E$ is called a $\alpha$-permanental process with kernel $(u(x, y): x, y \in E)$ if for every $x_{1}, \ldots, x_{n} \in E$ and $s_{1}, \ldots, s_{n} \geq 0$

$$
\mathbb{E} \exp \left\{-\sum_{j=1}^{n} s_{j} Y_{x_{j}}\right\}=|I+U S|^{-\alpha}
$$


where $U=\left(u\left(x_{i}, x_{j}\right): 1 \leq i, j \leq n\right)$ and $S=\operatorname{diag}\left(s_{1}, \ldots, s_{n}\right)$ are $n \times n$-matrices, and $\alpha>0$.

Hence, $Y_{x}$ 's are gamma distributed with shape parameter $\alpha$ and mean $\alpha u(x, x)$ and jointly they have a multivariate multivariate gamma distribution, as defined by (4.10). A prototype of a permanental process is a squared Gaussian processes, where $u(x, y)$ is the Gaussian covariance multiplied by 2 and $\alpha=1 / 2$.

For a fixed kernel $(u(x, y): x, y \in E)$, let $Y^{(\alpha)}=\left(Y_{x}^{(\alpha)}\right)_{x \in E}$ denote the corresponding $\alpha$-permanental process, if it exists. It is easy to see from (4.10) that if $Y^{(\alpha)}$ exists and is infinitely divisible for some $\alpha=\alpha_{0}>0$, then it does exist for every $\alpha$. Conversely, the existence of $Y^{(\alpha)}$ for every $\alpha>0$ implies that all $Y^{(\alpha)}$ are Poissonian infinitely divisible.

The importance of permanental processes comes also from their connection to Markov processes, as established by Eisenbaum and Kaspi [9]. They showed in [9], Theorem 3.1, that if $X=\left(X_{t}\right)_{t \geq 0}$ is a transient Markov process with a state space $E$ and 0-potential density $(u(x, y): x, y \in E)$ with respect to some reference measure, then for every $\alpha>0$ there exists a $\alpha$-permanental process $Y^{(\alpha)}=\left(Y_{x}^{(\alpha)}\right)_{x \in E}$ with kernel $u(x, y)$. Since $Y^{(\alpha)}=\left(Y_{x}^{(\alpha)}\right)_{x \in E}$ must be infinitely divisible and onedimensional marginals are nonnegative without drift, from Theorem 2.8 there is a Lévy measure $v$ on $\left(\mathbb{R}^{E}, \mathscr{B}^{E}\right)$ such that

$$
\mathbb{E} \exp \left\{-\sum_{j=1}^{n} s_{j} Y_{x_{j}}^{(\alpha)}\right\}=\exp \left[\int_{\mathbb{R}_{+}^{E}}\left(e^{-\sum_{j=1}^{n} s_{j} y\left(x_{j}\right)}-1\right) \alpha \nu(d y)\right],
$$

for all $x_{1}, \ldots, x_{n} \in E, s_{1}, \ldots, s_{n} \geq 0$, and $n \geq 1 . v$ is the Lévy measure of the 1-permanental process. Under some weak assumptions on $Y^{(\alpha)}$, such as its separability in probability, $v$ is also $\sigma$-finite; see Corollary 2.18. The canonical spectral representation of $Y^{(\alpha)}$ is of the form

$$
Y_{x}^{(\alpha)}=\int_{\mathbb{R}_{+}^{E}} y(x) N^{(\alpha)}(d y), \quad x \in E,
$$

where $N^{(\alpha)}$ is a Poisson random measure with intensity $\alpha \nu$.

To formulate the Dynkin isomorphism theorem, we need more ingredients. Recall, a transient Markov process $X=\left(X_{t}\right)_{t \geq 0}$ specified above. Assume that $X$ admits the local time $\left(L_{t}^{x}: x \in E, t \geq 0\right)$, which is normalized to satisfy $\mathbb{E}_{x}\left(L_{\infty}^{y}\right)=u(x, y)$. Fix $a \in E$ with $u(a, a)>0$, and let $\tilde{\mathbb{P}}_{a}$ be the probability under which the process $X$ starts at $a$ and is killed at its last visit to $a$. Then, for any measurable functional $F: \mathbb{R}^{E} \mapsto \mathbb{R}$,

$$
\mathbb{E} \tilde{\mathbb{E}}_{a}\left[F\left(\left(Y_{x}^{(\alpha)}+L_{\infty}^{x}\right)_{x \in E}\right)\right]=\mathbb{E}\left[F\left(\left(Y_{x}^{(\alpha)}\right)_{x \in E}\right) ; \frac{Y_{a}^{(\alpha)}}{\alpha u(a, a)}\right] .
$$

This identity is a version of the Dynkin isomorphism theorem due to Eisenbaum and Kaspi [9], Theorem 3.2. Here, we assume that the processes $Y^{(\alpha)}=\left(Y_{x}^{(\alpha)}\right)_{x \in E}$ 
and $L_{\infty}=\left(L_{\infty}^{x}\right)_{x \in E}$ depend on different coordinates of the product probability space under the product measure $\mathbb{P} \otimes \tilde{\mathbb{P}}_{a}$, so that $Y^{(\alpha)}$ and $L_{\infty}$ are independent.

To show that (4.13) fits the framework of Theorem 4.1, we will check that $\mathscr{L}\left(L_{\infty}\right) \ll \alpha \nu$. Indeed, by [15], Lemma 2.6.2, and direct computations as in [9], we have for every $x_{1}=a, x_{2}, \ldots, x_{n} \in E$ and $s_{1}, \ldots, s_{n} \geq 0$,

$$
\tilde{\mathbb{E}}_{a} \exp \left\{-\sum_{j=1}^{n} s_{j} L_{\infty}^{x_{j}}\right\}=\frac{1}{u(a, a)} \frac{\partial}{\partial s_{1}} \log |I+U S| .
$$

Combining (4.10), (4.11) and (4.14) we get

$$
\begin{aligned}
\tilde{\mathbb{E}}_{a} \exp \left\{-\sum_{j=1}^{n} s_{j} L_{\infty}^{x_{j}}\right\} & =-\frac{1}{\alpha u(a, a)} \frac{\partial}{\partial s_{1}} \log |I+U S|^{-\alpha} \\
& =-\frac{1}{\alpha u(a, a)} \frac{\partial}{\partial s_{1}}\left[\int_{\mathbb{R}^{E}}\left(e^{-\sum_{j=1}^{n} s_{j} y\left(x_{j}\right)}-1\right) \alpha v(d y)\right] \\
& =\frac{1}{u(a, a)} \int_{\mathbb{R}_{+}^{E}} e^{-\sum_{j=1}^{n} s_{j} y\left(x_{j}\right)} y(a) v(d y),
\end{aligned}
$$

which implies $\mathscr{L}\left(L_{\infty}\right) \ll \alpha \nu$. Now we will deduce (4.13) from Theorem 4.3(a). By the above,

$$
q(y):=\frac{d \mathscr{L}\left(L_{\infty}\right)}{d(\alpha \nu)}(y)=\frac{y(a)}{\alpha u(a, a)}, \quad y \in \mathbb{R}_{+}^{E}
$$

and from (4.12),

$$
N^{(\alpha)}(q)=\int_{\mathbb{R}_{+}^{E}} \frac{y(a)}{\alpha u(a, a)} N^{(\alpha)}(d y)=\frac{Y_{a}^{(\alpha)}}{\alpha u(a, a)} .
$$

Therefore, (4.3) gives (4.13). Moreover, since $Y_{a}^{(\alpha)}>0$, by (4.4) we also get

$$
\mathbb{E}\left[F\left(\left(Y_{x}^{(\alpha)}\right)_{x \in E}\right)\right]=\alpha u(a, a) \tilde{\mathbb{E}}_{a} \mathbb{E}\left[F\left(\left(Y_{x}^{(\alpha)}+L_{\infty}^{x}\right)_{x \in E}\right)\left(Y_{a}^{(\alpha)}+L_{\infty}^{a}\right)^{-1}\right] .
$$

Finally, notice that, while $\mathscr{L}\left(L_{\infty}\right)$ is absolutely continuous with respect to $v$, it is not equivalent to $v$. Indeed, the set $B=\left\{y \in \mathbb{R}_{+}^{E}: y(a)=0\right\}$ is a null set for $\mathscr{L}\left(L_{\infty}\right)$ under $\tilde{\mathbb{P}}_{a}$, but it is not a null set for $v$, even in the case when $E$ is a two-point set. Indeed, from Vere-Jones [27] we know that the Lévy measure of a two-dimensional permanental vector has singular components on the axes, except for the trivial totally correlated case, so that $v(B)>0$ in general. Nevertheless, path regularities of a permanental process transfer to $v$ by the transfer of regularity for Lévy measures (Theorem 3.4), and by the absolute continuity, they transfer to $L_{\infty}=\left(L_{\infty}^{x}\right)_{x \in E}$.

In Example 4.5, we have started with an infinitely divisible permanental process and showed that Dynkin's isomorphism (4.13) is a special case of Theorem 4.1. 
N. Eisenbaum [7], Lemma 3.1, made a surprising observation that any isomorphism of the type (4.13) implies the infinite divisibility of the process. We will reproduce this result in more detail and establish the form of Lévy measure in a general setting. We begin with random vectors.

LEMMA 4.6. Let $Y=\left(Y_{1}, \ldots, Y_{n}\right)$ be a nonnegative random vector with $\theta_{i}=$ $\mathbb{E}\left(Y_{i}\right) \in(0, \infty)$. The following are equivalent:

(i) $Y$ is infinitely divisible;

(ii) For every $k \leq n$, there exists a vector of nonnegative random variables $Z^{k}=$ $\left(Z_{1}^{k}, \ldots, Z_{n}^{k}\right)$ independent of $Y$ such that for any bounded measurable functional $F: \mathbb{R}^{n} \mapsto \mathbb{R}:$

$$
\mathbb{E} F\left(Y+Z^{k}\right)=\mathbb{E}\left[F(Y) ; \theta_{k}^{-1} Y_{k}\right]
$$

Moreover, if (ii) holds, then $Y$ has the Lévy measure $v$ on $\mathbb{R}_{+}^{n}$ of the form

$$
v(d y)=\sum_{k=1}^{n} \theta_{k} \mathbf{1}_{A_{k}}(y) y_{k}^{-1} \mathscr{L}\left(Z^{k}\right)(d y),
$$

where $A_{k}=\left\{y \in \mathbb{R}_{+}^{n}: y_{1}=\cdots=y_{k-1}=0, y_{k}>0\right\}$. The drift of $Y$ equals

$$
c=\left(\theta_{1} \mathbb{P}\left(Z_{1}^{1}=0\right), \ldots, \theta_{n} \mathbb{P}\left(Z_{n}^{n}=0\right)\right) .
$$

Furthermore, the law of each $Z^{k}$ is determined the law of $Y, k=1, \ldots, n$.

Now we characterize processes satisfying the abstract version of Dynkin's isomorphism.

Proposition 4.7. Let $Y=\left(Y_{t}\right)_{t \in T}$ be a nonnegative process with $\theta(t)=$ $\mathbb{E} Y_{t}<\infty$ for every $t \in T$. Suppose that for every $s \in T$ having $\theta(s)>0$, there exists a stochastic process $Z^{s}=\left(Z_{t}^{s}\right)_{t \in T}$ independent of $Y$ such that for any measurable functional $F: \mathbb{R}^{T} \mapsto \mathbb{R}$ :

$$
\mathbb{E}\left[F\left(\left(Y_{t}+Z_{t}^{s}\right)_{t \in T}\right)\right]=\mathbb{E}\left[F\left((Y)_{t \in T}\right) ; \theta(s)^{-1} Y_{s}\right]
$$

Then the process $Y$ is infinitely divisible. If, in addition, $Y$ is separable in probability with a separant $T_{0}=\left(s_{k}\right)_{k \geq 1}$, then the Lévy measure $v$ of $Y$ is of the form

$$
v=\sum_{k \geq 1} \mathbf{1}_{A_{k}} v_{k}
$$

where $A_{k}=\left\{y \in \mathbb{R}_{+}^{T}: y\left(s_{i}\right)=0 \forall i<k, y\left(s_{k}\right)>0\right\}$ are disjoint and $v_{k}$ are Lévy measures given by

$$
v_{k}(d y)=\theta\left(s_{k}\right) y\left(s_{k}\right)^{-1} \mathscr{L}\left(Z^{s_{k}}\right)(d y), \quad k=1,2, \ldots
$$

The drift of $Y$ is given by $c=\left(\theta(t) \mathbb{P}\left(Z_{t}^{t}=0\right): t \in T\right)$. 
Moreover, any nonnegative finite mean infinitely divisible process $Y=\left(Y_{t}\right)_{t \in T}$ satisfies (4.18) with $Z^{s}=\left(Z_{t}^{s}\right)_{t \in T}$ determined by

$$
\mathscr{L}\left(Z^{s}\right)(d y)=\frac{c(s)}{\theta(s)} \delta_{0_{T}}(d y)+\frac{y(s)}{\theta(s)} v(d y),
$$

where $c$ and $v$ are the drift and Lévy measure of $Y$, respectively, and $\theta(s)=\mathbb{E} Y_{s}>$ 0 .

REMARK 4.8. Consider a separable in probability process $Y$ of Proposition 4.7 with Lévy measure $v$ given by (4.19). Let $a \in T$ and $\theta(a)>0$. We can always include $a$ in $T_{0}$ and assume that $s_{1}=a$. If $Z_{a}^{a}>0$ a.s., then $\mathscr{L}\left(Z^{a}\right) \ll$ $v_{1} \ll v$, so that Theorem 4.3(a) gives (4.18). If $\mathbb{P}\left(Z_{a}^{a}=0\right)>0$, then $\mathscr{L}\left(Z^{a}\right) \ll v$ but $\mathscr{L}\left(Z^{a}\right) \ll v+\delta_{0_{T}}$, so that Theorem 4.3(b) gives (4.18) in this case.

REMARK 4.9. If $Y=\left(Y_{1}, Y_{2}\right)$ satisfies (4.15), then Lévy measure of $Y$ has the form

$$
\begin{aligned}
v(d y)= & \theta_{1} \mathbf{1}_{\left\{y_{1}>0, y_{2}=0\right\}} y_{1}^{-1} \mathscr{L}\left(Z^{1}\right)(d y)+\theta_{2} \mathbf{1}_{\left\{y_{1}=0, y_{2}>0\right\}} y_{2}^{-1} \mathscr{L}\left(Z^{2}\right)(d y) \\
& +\theta_{1} \mathbf{1}_{\left\{y_{1}>0, y_{2}>0\right\}} y_{1}^{-1} \mathscr{L}\left(Z^{1}\right)(d y) .
\end{aligned}
$$

This formula may shed some light on the form of Lévy measure of a 2-dimensional permanental vector in Vere-Jones [27], which has positive masses on the axes.

Isomorphism identities can also be useful for Lévy processes. We begin with a corollary to Theorem 4.4 .

COROLlary 4.10. Let $X=\left(X_{t}\right)_{t \geq 0}$ be a Lévy process such that $\mathbb{E} e^{i u X_{t}}=$ $e^{t K(u)}$, where

$$
K(u)=-\frac{1}{2} \sigma^{2} u^{2}+\int_{\mathbb{R}}\left(e^{i u x}-1-i u \llbracket x \rrbracket\right) \rho(d x)+i c u .
$$

Let $q: \mathbb{R}_{+} \times \mathbb{R} \mapsto \mathbb{R}_{+}$be a measurable function such that $\int_{\mathbb{R}_{+} \times \mathbb{R}} q(r, v) d r \rho(d v)=$ 1. Then for any measurable functional $F: \mathbb{R}^{[0, \infty)} \mapsto \mathbb{R}$

$$
\mathbb{E} \int_{\mathbb{R}_{+} \times \mathbb{R}} F\left(\left(X_{t}+\mathbf{1}_{\{r \leq t\}} v\right)_{t \geq 0}\right) q(r, v) d r \rho(d v)=\mathbb{E}\left[F\left(\left(X_{t}\right)_{t \geq 0}\right) ; g(X)\right],
$$

where $g(X)=\sum_{\left\{r>0: \Delta X_{r} \neq 0\right\}} q\left(r, \Delta X_{r}\right)$ and $\Delta X_{r}=X_{r}-X_{r-}$. Conversely,

$$
\begin{aligned}
& \mathbb{E}\left[F\left(\left(X_{t}\right)_{t \geq 0}\right) ; g(X)>0\right] \\
& \quad=\int_{\mathbb{R}_{+} \times \mathbb{R}} \mathbb{E}\left[F\left(\left(X_{t}+\mathbf{1}_{\{r \leq t\}} v\right)_{t \geq 0}\right) ;(g(X)+q(r, v))^{-1}\right] q(r, v) d r \rho(d v) .
\end{aligned}
$$

Moreover, $g(X)>0$ a.s. if $\int_{\mathbb{R}_{+} \times \mathbb{R}} \mathbf{1}\{q(r, v)>0\} d r \rho(d v)=\infty$. 
PROOF. This is a direct application of Theorem 4.4. Indeed, has the Lévy-Itô decomposition

$$
X_{t}=G_{t}+\int_{\mathbb{R}_{+} \times \mathbb{R}} \mathbf{1}_{\{r \leq t\}} v(N(d r, d v)-\chi(v) d r \rho(d v))+c t,
$$

where $N=\sum_{\left\{r: \Delta X_{r} \neq 0\right\}} \delta_{\left(r, \Delta X_{r}\right)}$. Hence

$$
N(q)=\int_{\mathbb{R}_{+} \times \mathbb{R}} q(r, v) N(d r, d v)=\sum_{\left\{r>0: \Delta X_{r} \neq 0\right\}} q\left(r, \Delta X_{r}\right):=g(X),
$$

as desired.

The next example specifies a set of admissible random translations for a Poisson process.

EXAMPLE 4.11. Let $Y=\left\{Y_{t}\right\}_{t \geq 0}$ be a Poisson process with rate $\lambda>0$ and let $Z_{t}=\mathbf{1}_{[\zeta, \infty)}(t), t \geq 0$, where $\zeta>0$ is a random variable with density $h$ and independent of $Y$. Then $\mathscr{L}(Y+Z) \ll \mathscr{L}(Y)$ and

$$
\mathbb{E}\left[F\left(\left(Y_{t}+Z_{t}\right)_{t \geq 0}\right)\right]=\mathbb{E}\left[F\left(\left(Y_{t}\right)_{t \geq 0}\right) ; g(Y)\right],
$$

where $g(Y)=\lambda^{-1} \int_{0}^{\infty} h d Y$. Conversely,

$$
\mathbb{E}\left[F\left(\left(Y_{t}\right)_{t \geq 0}\right) ; g(Y)>0\right]=\mathbb{E}\left[F\left(\left(Y_{t}+Z_{t}\right)_{t \geq 0}\right) ;\left(g(Y)+\lambda^{-1} h(\zeta)\right)^{-1}\right] .
$$

Moreover, $g(Y)>0$ a.s. if $\int_{\mathbb{R}_{+}} 1\{h(r)>0\} d r=\infty$, in which case $\mathscr{L}(Y+Z)$ and $\mathscr{L}(Y)$ are equivalent.

PROOF. It follows from Corollary 4.10 for $q(r, v)=\lambda^{-1} h(r)$ and $\rho=\lambda \delta_{1}$.

In the previous example, function $q$ depended only on time variable. Now we consider the case when $q$ depends only on the space variable.

EXAMPLE 4.12. Let $X=\left(X_{t}\right)_{t \geq 0}$ be a Lévy process as in Corollary 4.10. Let $q_{1}: \mathbb{R} \mapsto \mathbb{R}_{+}$be such that $\int_{\mathbb{R}} q_{1}(v) \rho(d v)=1$. Consider a fixed time horizon $h>0$, so that $T=[0, h]$ and let

$$
X_{t}=G_{t}+\int_{[0, h] \times \mathbb{R}} \mathbf{1}_{\{r \leq t\}} v(N(d r, d v)-\chi(v) d r \rho(d v))+c t, \quad t \in[0, h] .
$$

To apply Theorem 4.4 , take $q(r, v)=h^{-1} q_{1}(v)$, so that $\int_{[0, h] \times \mathbb{R}} q(r, v) d r \rho(d v)=$ 1 , and compute

$$
N(q)=\int_{[0, h] \times \mathbb{R}} q(r, v) N(d r, d v)=h^{-1} \int_{[0, h] \times \mathbb{R}} q_{1}(v) N(d r, d v):=h^{-1} W_{h},
$$


where $W=\left(W_{h}\right)_{h \geq 0}$ is a subordinator with Lévy measure $\rho_{1}$ given by $\rho_{1}(x, \infty)=$ $\rho\left(q_{1}^{-1}(x, \infty)\right), x \geq 0$. Then, for every measurable $F: \mathbb{R}^{[0, h]} \mapsto \mathbb{R}$,

$$
\mathbb{E} \int_{\mathbb{R}} F\left(\left(X_{t}+\mathbf{1}_{\{r \leq t\}} v\right)_{t \in[0, h]}\right) q_{1}(v) \rho(d v)=h^{-1} \mathbb{E}\left[F\left(\left(X_{t}\right)_{t \in[0, h]}\right) ; W_{h}\right]
$$

and

$$
\begin{aligned}
& \mathbb{E}\left[F\left(\left(X_{t}\right)_{t \in[0, h]}\right) ; W_{h}>0\right] \\
& \quad=h \int_{\mathbb{R}} \mathbb{E}\left[F\left(\left(X_{t}+\mathbf{1}_{\{r \leq t\}} v\right)_{t \in[0, h]}\right) ;\left(W_{h}+q_{1}(v)\right)^{-1}\right] q_{1}(v) \rho(d v) .
\end{aligned}
$$

To illustrate usefulness of these formulas, in the next corollary we give an alternative proof to a known fact on the behavior of the distributions of the Lévy process at the origin. Actually, we prove a more general version of this fact (see, e.g., [24], Corollary 8.9), and the last statement of the corollary seems to be new.

Proposition 4.13. Let $X=\left(X_{t}\right)_{t \geq 0}$ be a Lévy process and let $\rho$ be the Lévy measure of $X_{1}$. Let $f: \mathbb{R} \mapsto \mathbb{R}$ be a bounded function continuous on a set of full $\rho$-measure such that $f(x)=o\left(x^{2}\right)$ as $x \rightarrow 0$ [or $f(x)=O\left(x^{2}\right)$ when $X$ has no Gaussian component]. Then

$$
\lim _{h \rightarrow 0} h^{-1} \mathbb{E} f\left(X_{h}\right)=\int_{\mathbb{R}} f(v) \rho(d v) .
$$

If $X$ is a subordinator, then the assumption of continuity can be weakened to the right-continuity.

COROLLARY 4.14. If $X$ is a Lévy process then for any $\rho$-continuity set $B$ with $0 \notin \bar{B}$, we have $\lim _{h \rightarrow 0} h^{-1} \mathbb{P}\left(X_{h} \in B\right)=v(B)$. If in addition $X$ is a subordinator, then for every $r>0$ we have $\lim _{h \rightarrow 0} h^{-1} \mathbb{P}\left(X_{h} \in[r, \infty)\right)=v([r, \infty))$.

PROOF. In the first part of the corollary, we take $f(x)=\mathbf{1}_{B}(x)$ and in the second part we take $f(x)=\mathbf{1}[r, \infty)(x)$, and apply the above.

5. Series representations and isomorphism identities. Here, we will show how representations of Lévy measures lead to series representations of Poissonian infinitely divisible processes. This method of constructing series representations was initiated in [20] and further developed in [22].

THEOREM 5.1. Let $Y=\left(Y_{t}\right)_{t \in T}$ be a Poissonian infinitely divisible process with the generating triplet $(0, v, b)$. Let $V=\left(V_{t}\right)_{t \in T}$ be a representation of $v$ on a $\sigma$-finite measure space $(S, \mathscr{S}, n)$. Consider a probability measure $n^{(1)}$ equivalent to $n$, so that $n^{(1)}(d s)=g(s) n(d s)$ for some $g>0$ n-a.e. Let $\left(\xi_{j}\right)_{j \in \mathbb{N}}$ be an i.i.d. sequence of random elements in $S$ with the common distribution $n^{(1)}$ and let $\left(\Gamma_{j}\right)_{j \in \mathbb{N}}$ be a sequence of partial sums of i.i.d. mean-one exponential random variables independent of the sequence $\left(\xi_{j}\right)_{j \in \mathbb{N}}$. Then: 
(i) For every $t \in T$, the series

$$
S_{t}^{(0)}:=\sum_{j=1}^{\infty} V_{t}\left(\xi_{j}\right) \mathbf{1}\left\{g\left(\xi_{j}\right) \leq \Gamma_{j}^{-1}\right\}
$$

converges a.s. if and only if the limit

$$
c(t):=\lim _{j \rightarrow \infty} \int_{S} \llbracket V_{t}(s) \rrbracket(j g(s) \wedge 1) n(d s) \quad \text { exists. }
$$

If (5.2) holds, then

$$
\left(Y_{t}\right)_{t \in T} \stackrel{d}{=}\left(S_{t}^{(0)}+b(t)-c(t)\right)_{t \in T} .
$$

In particular, if $Y_{t} \geq 0$, or more generally, if $\int_{\mathbb{R}^{T}}|x(t)| \wedge 1 v(d x)<\infty$, then (5.2) holds.

(ii) For every $t \in T$, the centered series

$$
S_{t}:=\sum_{j=1}^{\infty}\left[V_{t}\left(\xi_{j}\right) \mathbf{1}\left\{g\left(\xi_{j}\right) \leq \Gamma_{j}^{-1}\right\}-c_{j}(t)\right]
$$

converges a.s. and

$$
\left(Y_{t}\right)_{t \in T} \stackrel{d}{=}\left(S_{t}+b(t)\right)_{t \in T}
$$

where

$$
c_{j}(t)=\int_{S} \llbracket V_{t}(s) \rrbracket\{(j g(s) \wedge 1)-((j-1) g(s) \wedge 1)\} n(d s) .
$$

Under (5.2), $\sum_{j=1}^{\infty} c_{j}(t)=c(t)$, so that $S_{t}=S_{t}^{(0)}-c(t)$.

PROOF. This proof is a routine application of Theorem 4.1 [22], and thus it is omitted.

REMARK 5.2. (a) If $Y$ is separable in probability and defined on a rich enough probability space (see Theorem 3.2), then proceeding as in [22], we can choose $V_{j}, \Gamma_{j}$ on the same probability space as $Y$ such that (5.3) and (5.5) hold not only in distribution but also almost surely.

(b) If sample paths of $Y$ belong to a separable Banach space, then the pointwise convergent series (5.1) and (5.4) converge a.s. in the norm of that Banach space. Such conclusion is generally false when sample paths of $Y$ belong to a nonseparable Banach space. An exception is the Skorohod space under the uniform topology, which is not separable. However, in such space the series converge uniformly a.s.; see [3].

(c) There is some analogy between series expansions of Poissonian infinitely divisible process, such as in Theorem 5.1, and Karhunen-Loève series representation of Gaussian processes. Exploring this analogy, one has the corresponding results for the oscillation and zero-one laws of Poissonian infinitely divisible process; see [4] and [21]. 
EXAMPLE 5.3 (Feller diffusions). Let $Z=\left(Z_{t}\right)_{t \in T}$ be a Feller diffusion, as in Example 2.25. Recall that $V_{t}=L_{\infty}^{\kappa t}, t \geq 0$ is a representation of the Lévy measure of $Z$ on $(S, n)=\left(U_{+}, a n_{+}\right)$. We will now give a probability measure $n^{(1)}$ equivalent to $a n_{+}$. Let $R(u)$ denote the length of an excursion $u \in U_{+}$. It is well known that

$$
n_{+}\{u: R(u)>x\}=\frac{1}{\sqrt{2 \pi}} x^{-1 / 2} ;
$$

see, for example, [19], Chapter 12, Proposition 2.8. Let $f: \mathbb{R}_{+} \mapsto \mathbb{R}_{+}$be such that $f(x)=0$ only for $x=0$ and

$$
\frac{1}{\sqrt{2 \pi}} \int_{0}^{\infty} f^{\prime}(x) x^{-1 / 2} d x=1 .
$$

Put $n^{(1)}(d u):=f(R(u)) n_{+}(d u)$. Then

$$
\int_{U_{+}} f(R(u)) n_{+}(d u)=\int_{0}^{\infty} f^{\prime}(x) n_{+}\{u: R(u)>x\} d x=1,
$$

so that $n^{(1)}$ is a probability measure such that $g(u):=\frac{d n^{(1)}}{d\left(a n_{+}\right)}(u)=a^{-1} f(R(u))$. Now we will apply Theorem 5.1(a). Let $\left(\xi_{j}\right)_{j \in \mathbb{N}}$ be an i.i.d. sequence of random elements in $U_{+}$with the common distribution $f(R) d n_{+}$and let $\left(\Gamma_{j}\right)_{j \in \mathbb{N}}$ be a sequence of partial sums of i.i.d. mean-one exponential random variables independent of the sequence $\left(\xi_{j}\right)_{j \in \mathbb{N}}$. Then

$$
Z_{t} \stackrel{d}{=} \sum_{j=1}^{\infty} L_{\infty}^{\kappa t}\left(\xi_{j}\right) \mathbf{1}\left\{f\left(R\left(\xi_{j}\right)\right) \leq a \Gamma_{j}^{-1}\right\}, \quad t \geq 0
$$

in the sense of equality of finite dimensional distributions. By Remark 5.2(b), the convergence holds also a.s. uniformly in $t$ on finite intervals. Let us take $f(x)=$ $\sqrt{\frac{\pi}{2}}(x \wedge 1)$ for concreteness. Then the above formula becomes

$$
Z_{t} \stackrel{d}{=} \sum_{j=1}^{\infty} L_{\infty}^{\kappa t}\left(\xi_{j}\right) \mathbf{1}\left\{R\left(\xi_{j}\right) \wedge 1 \leq(2 / \pi)^{1 / 2} a \Gamma_{j}^{-1}\right\}, \quad t \geq 0
$$

This formula says that a Feller diffusion is the series of randomly trimmed total accumulated local times taken at the level $\kappa t, t \geq 0$ from an infinite sample of Brownian excursions. This sample is taken according to the density $(\pi / 2)^{1 / 2}(R \wedge$ 1) with respect to $n_{+}$.

Along similar lines, we obtain series representations of squared Bessel process.

EXAMPLE 5.4 (Squared Bessel processes). Let $Y=\left(Y_{t}\right)_{t \in T}$ be a squared $\beta$ dimensional Bessel process starting from 0 and $\beta>0$, as in Example 2.24. Recall that $V_{t}=L_{\infty}^{t-(\cdot)}, t \geq 0$ is a representation of the Lévy measure of $Y$ on $(S, n)=$ 
$\left(\mathbb{R}_{+} \times U_{+}, \beta \lambda \otimes n_{+}\right)$. Recall that $L_{\infty}^{a}(u)=0$ when $a \leq 0$. Let $f$ and $R$ be as in Example 5.3. Put

$$
n^{(1)}(d r, d u)=\left(\beta e^{-\beta r} d r\right) \otimes f(R(u)) n_{+}(d u), \quad r>0, u \in U_{+} .
$$

$n^{(1)}$ is a probability measure equivalent to $\beta \lambda \otimes n_{+}$. Let $\left\{\eta_{n}\right\}$ be an i.i.d. sequence of exponential random variables with parameter $\beta$, let $\left(\xi_{j}\right)_{j \in \mathbb{N}}$ be an i.i.d. sequence of random elements in $U_{+}$with the common distribution $f(R) d n_{+}$and let $\left(\Gamma_{j}\right)_{j \in \mathbb{N}}$ be a sequence of partial sums of i.i.d. mean-one exponential random variables. Assume that these sequences are independent of each other. We compute

$$
g(r, u)=\frac{d n^{(1)}}{d n}(d r, d u)=e^{-\beta r} f(R(u)) .
$$

By Theorem 5.1(i) and Remark 5.2(b),

$$
Y_{t} \stackrel{d}{=} \sum_{j=1}^{\infty} L_{\infty}^{t-\eta_{j}}\left(\xi_{j}\right) \mathbf{1}\left\{e^{-\beta \eta_{j}} f\left(R\left(\xi_{j}\right)\right) \leq \Gamma_{j}^{-1}\right\}, \quad t \geq 0
$$

in the sense of equality of finite dimensional distributions and the series converges uniformly a.s. Again, choosing a specific $f$, as at the end of Example 5.3, may give more insight into this representation.

Series representations of Lévy processes have been considered in many places, so we will only sketch representations resulting from Theorem 5.1.

EXAMPLE 5.5 (Lévy process). Let $Y=\left(Y_{t}\right)_{t \geq 0}$ be a Poissonian-Lévy process, as in Example 2.23. Then $V_{t}(r, v)=\mathbf{1}_{\{r \leq t\}} v$ is a representation of the Lévy measure on $\left(\mathbb{R}_{+} \times \mathbb{R}, \lambda \otimes \rho\right)$. Choose an arbitrary probability measure $n^{(1)}$ on $\mathbb{R}_{+} \times \mathbb{R}$ that is equivalent to $\lambda \otimes \rho$ and put $g(r, v)=\frac{d n^{(1)}}{d(\lambda \otimes \rho)}(r, v)$. Let $\xi_{j}=\left(\eta_{j}, v_{j}\right)$, $j \in \mathbb{N}$ be i.i.d. random variables with the common density $g$ with respect to $\lambda \otimes \rho$. Then by Theorem 5.1(ii) and Remark 5.2(b),

$$
Y_{t} \stackrel{d}{=} \sum_{j=1}^{\infty}\left[\mathbf{1}_{\left\{\eta_{j} \leq t\right\}} v_{j} \mathbf{1}\left\{g\left(\eta_{j}, v_{j}\right) \leq \Gamma_{j}^{-1}\right\}-c_{t}(j)\right]+b_{t}
$$

in the sense of equality of finite dimensional distributions and the series converges uniformly almost surely.

THEOREM 5.6 (Series form of isomorphism). Under notation of Theorem 5.1, consider a Poissonian infinitely divisible process $Y=\left(Y_{t}\right)_{t \in T}$ having Lévy measure $v$ and given by (5.4)

$$
Y_{t}=\sum_{j=1}^{\infty}\left[V_{t}\left(\xi_{j}\right) \mathbf{1}\left\{g\left(\xi_{j}\right) \leq \Gamma_{j}^{-1}\right\}-c_{j}(t)\right]+b(t)
$$


where $V=\left(V_{t}\right)_{t \in T}$ is a representation of $v$ on $(S, \mathscr{S}, n)$ and $g=\frac{d \mathscr{L}\left(\xi_{1}\right)}{d v}>0$. Let $\xi_{0}$ be a random variable in $S$ independent of $\left(\xi_{j}, \Gamma_{j}\right)_{j \in \mathbb{N}}$ such that $\mathscr{L}\left(\xi_{0}\right) \ll$ $\mathscr{L}\left(\xi_{1}\right)$ and let $q=\frac{d \mathscr{L}\left(\xi_{o}\right)}{d \nu}$. Then for any measurable functional $F: \mathbb{R}^{T} \mapsto \mathbb{R}$

$$
\mathbb{E}\left[F\left(\left(V_{t}\left(\xi_{0}\right)+Y_{t}\right)_{t \in T}\right)\right]=\mathbb{E}\left[F\left(\left(Y_{t}\right)_{t \in T}\right) ; Q\right],
$$

where

$$
Q=\sum_{j=1}^{\infty} q\left(\xi_{j}\right) \mathbf{1}\left\{g\left(\xi_{j}\right) \leq \Gamma_{j}^{-1}\right\}
$$

Conversely,

$$
\mathbb{E}\left[F\left(\left(Y_{t}\right)_{t \in T}\right) ; Q>0\right]=\mathbb{E} \int_{S}\left[F\left(\left(V_{t}(s)+Y_{t}\right)_{t \in T}\right) ;(Q+q(s))^{-1}\right] q(s) n(d s) .
$$

Moreover, $Q>0$ a.s. provided $n\{s: q(s)>0\}=\infty$.

\section{Proofs.}

Proof Of Lemma 2.3. Clearly, the left-hand side is greater or equal than the right-hand side in (2.2). To prove the reverse inequality, take $A \in \mathscr{B}^{T}$. Since

$$
v_{*}\left(A \backslash 0_{T}\right)=\sup \left\{v(D): D \subset A \backslash 0_{T}, D \in \mathscr{B}^{T}\right\},
$$

there exists an $A_{0} \in \mathscr{B}^{T}$ with $A_{0} \subset A \backslash 0_{T}$ such that $v\left(A_{0}\right)=v_{*}\left(A \backslash 0_{T}\right)$. From the structure of $\mathscr{B}^{T}, A_{0}=\pi_{U}^{-1}(B)$ for some set $U \in \hat{T}_{c}$ and $B \in \mathscr{B}^{U}$, with $0_{U} \notin B$. Let $J_{n} \in \hat{T}$ be such that $J_{n} \uparrow U$. By the continuity of $v$ from below,

$$
\begin{aligned}
v_{*}\left(A \backslash 0_{T}\right) & =v\left(A_{0}\right)=v\left(A_{0} \backslash \pi_{U}^{-1}\left(0_{U}\right)\right)=\lim _{n \rightarrow \infty} v\left(A_{0} \backslash \pi_{J_{n}}^{-1}\left(0_{J_{n}}\right)\right) \\
& \leq \sup _{J \in \hat{T}} v\left(A \backslash \pi_{J}^{-1}\left(0_{J}\right)\right),
\end{aligned}
$$

which establishes (2.2).

Since the measures $v\left(\cdot \backslash \pi_{J}^{-1}\left(0_{J}\right)\right), J \in \hat{T}$ are increasing as $J$ 's are increasing and $\hat{T}$ is a directed set under the inclusion,

$$
\nu^{0}(\cdot)=\sup _{J \in \hat{T}} v\left(\cdot \backslash \pi_{J}^{-1}\left(0_{J}\right)\right)
$$

is a measure. If $0_{T} \notin A \in \mathscr{B}^{T}$, then

$$
v^{0}(A)=v_{*}\left(A \backslash 0_{T}\right)=v_{*}(A)=v(A),
$$

which gives (2.3). Using (2.2)-(2.3), we get for every $A \in \mathscr{B}^{T}$

$$
\left(v^{0}\right)_{*}\left(A \backslash 0_{T}\right)=\sup _{J \in \hat{T}} v^{0}\left(A \backslash \pi_{J}^{-1}\left(0_{J}\right)\right)=\sup _{J \in \hat{T}} v\left(A \backslash \pi_{J}^{-1}\left(0_{J}\right)\right)=v^{0}(A),
$$

which establishes (L2). Finally, if $v$ satisfies (L1), then so does $v^{0}$. 
Proof of Lemma 2.4. (L2) $\Rightarrow$ (a). Let $T_{0} \in \hat{T}_{c}$. By (L2) and (2.2), there exists an increasing sequence $J_{n} \in \hat{T}$ such that

$$
v\left\{x: x_{T_{0}}=0\right\}=\lim _{n \rightarrow \infty} v\left\{x: x_{T_{0}}=0, x_{J_{n}} \neq 0\right\} .
$$

Therefore, $T_{1}=\bigcup J_{n}$ satisfies (a).

(a) $\Rightarrow$ (b). Let $J_{n}$ be as above. (a) implies that for some $n, v\left\{x: x_{T_{0}}=0, x_{J_{n}} \neq\right.$ $0\}>0$. Hence (2.4) holds for some $t \in J_{n}$.

(b) $\Rightarrow$ (c). If (2.1) and (2.4) do not hold, then we have a contradiction with (b).

(c) $\Rightarrow$ (L2). Since by Remark 2.2, (2.1) implies (L2), we only need to consider the second part of the alternative in (c); that is, we assume that (2.4) holds for any set $T_{0} \in \hat{T}_{c}$. Let $A \in \mathscr{B}^{T}$. (L2) obviously holds when $0_{T} \notin A$, so we consider the case $O_{T} \in A$. Using (2.2), as in the first implication of this proof, we infer that $v_{*}\left(A \backslash 0_{T}\right)=v\left(A \backslash\left\{x: x_{T_{1}}=0\right\}\right)$ for some $T_{1} \in \hat{T}_{c}$. There is also a countable set $T_{0} \supset T_{1}$ and $B \in \mathscr{B}^{T_{0}}$ such that $A=\left\{x: x_{T_{0}} \in B\right\}$. Let $t \notin T_{0}$ be such that $v\left\{x: x_{T_{0}}=0, x(t) \neq 0\right\}=\alpha>0$. Since $v_{*}\left(A \backslash 0_{T}\right)=v\left(A \backslash\left\{x: x_{T_{1}}=0\right\}\right)$ holds also for any larger set in place of $T_{1}$, we get

$$
\begin{aligned}
v_{*}\left(A \backslash 0_{T}\right) & =v\left(A \backslash\left\{x: x_{T_{0} \cup\{t\}}=0\right\}\right) \\
& =v\left(A \backslash\left\{x: x_{T_{0}}=0\right\}\right)+v\left(A \cap\left\{x: x_{T_{0}}=0\right\} \cap\{x: x(t) \neq 0\}\right) \\
& =v\left(A \backslash\left\{x: x_{T_{0}}=0\right\}\right)+v\left(\left\{x: x_{T_{0}}=0, x(t) \neq 0\right\}\right) \\
& =v_{*}\left(A \backslash 0_{T}\right)+\alpha,
\end{aligned}
$$

where in the third equality we used that $0_{T} \in A$. The above computation shows that $v_{*}\left(A \backslash 0_{T}\right)=\infty$, in which case (L2) trivially holds. The proof is complete.

PROOF. Proof of Theorem 2.8 The proof of Theorem 2.8 is divided into four steps.

Step 1. Let $J \in \hat{T}$ and $\varepsilon>0$ be fixed. Set $U=\left\{y \in \mathbb{R}^{J}: \max _{t \in J}\left|y_{t}\right|>\varepsilon\right\}$ and define a family of measures $\lambda_{I}^{J, \varepsilon}$ on $\left(\mathbb{R}^{I}, \mathscr{B}^{I}\right)$ by

$$
\lambda_{I}^{J, \varepsilon}(B)=v_{K}\left(\pi_{K I}^{-1}(B) \cap \pi_{K J}^{-1}(U)\right), \quad B \in \mathscr{B}^{I},
$$

where $I \in \hat{T}$ and $K=I \cup J$. We will show that there exists a finite measure $\lambda^{J, \varepsilon}$ on $\left(\mathbb{R}^{T}, \mathscr{B}^{T}\right)$ such that

$$
\lambda^{J, \varepsilon} \circ \pi_{I}^{-1}=\lambda_{I}^{J, \varepsilon} \quad \text { for all } I \in \hat{T} .
$$

First, observe that all measures $\lambda_{I}^{J, \varepsilon}, I \in \hat{T}$, have equal finite mass. Indeed, by (c3) and the fact that $v_{J}$ is a Lévy measure on $\mathbb{R}^{J}$

$$
\lambda_{I}^{J, \varepsilon}\left(\mathbb{R}^{I}\right)=v_{K}\left(\pi_{K J}^{-1}(U)\right)=v_{J}(U)<\infty .
$$


Next, we will show that $\left\{\lambda_{I}^{J, \varepsilon}: I \in \hat{T}\right\}$ is a projective system. Take $I_{1} \subset I_{2} \in \hat{T}$ and put $K_{1}=J \cup I_{1}, K_{2}=J \cup I_{2}$. We have for every $B \in \mathscr{B}^{I_{1}}$,

$$
\begin{aligned}
\lambda_{I_{2}}^{J, \varepsilon}\left(\pi_{I_{2}, I_{1}}^{-1}(B)\right) & =v_{K_{2}}\left(\pi_{K_{2}, I_{2}}^{-1}\left(\pi_{I_{2}, I_{1}}^{-1}(B)\right) \cap \pi_{K_{2}, J}^{-1}(U)\right) \\
& =v_{K_{2}}\left(\pi_{K_{2}, I_{1}}^{-1}(B) \cap \pi_{K_{2}, J}^{-1}(U)\right) \\
& =v_{K_{2}}\left(\pi_{K_{2}, K_{1}}^{-1}\left(\pi_{K_{1}, I_{1}}^{-1}(B) \cap \pi_{K_{1}, J}^{-1}(U)\right)\right) \\
& =v_{K_{1}}\left(\pi_{K_{1}, I_{1}}^{-1}(B) \cap \pi_{K_{1}, J}^{-1}(U)\right)=\lambda_{I_{1}}^{J, \varepsilon}(B) .
\end{aligned}
$$

In the fourth equality, we used (c3) as $\pi_{K_{1}, I_{1}}^{-1}(B) \cap \pi_{K_{1}, J}^{-1}(U)$ does not contain the origin of $\mathbb{R}^{K_{1}}$. By Kolmogorov's extension theorem, there exists a finite measure $\lambda^{J, \mathcal{E}}$ on $\left(\mathbb{R}^{T}, \mathscr{B}^{T}\right)$ satisfying (6.2).

Step 2. Define

$$
\nu(A)=\sup _{J \in \hat{T}, \varepsilon>0} \lambda^{J, \varepsilon}(A), \quad A \in \mathscr{B}^{T} .
$$

Then $v$ is a measure satisfying (2.7).

First, we observe from (6.1) that for every $I \in \hat{T}, \lambda_{I}^{J_{1}, \varepsilon_{1}} \leq \lambda_{I}^{J_{2}, \varepsilon_{2}}$ whenever $J_{1} \subset$ $J_{2}$ and $\varepsilon_{1} \geq \varepsilon_{2}$. This implies, in conjunction with (6.2), that $\lambda^{J_{1}, \varepsilon_{1}}(A) \leq \lambda^{J_{2}, \varepsilon_{2}}(A)$ for every $\bar{A}$ from the algebra of cylinders, $A \in \pi_{I}^{-1}\left(\mathscr{B}^{I}\right), I \in \hat{T}$. By the monotone class argument, we obtain

$$
\lambda^{J_{1}, \varepsilon_{1}} \leq \lambda^{J_{2}, \varepsilon_{2}} \quad \text { when } J_{1} \subset J_{2} \text { and } \varepsilon_{1} \geq \varepsilon_{2} .
$$

We will now check that $v$ is a measure. For any pairwise disjoint sets $A_{n} \in \mathscr{B}^{T}$, we have

$$
\begin{aligned}
v\left(\bigcup_{i=1}^{\infty} A_{i}\right) & =\sup _{J \in \hat{T}, \varepsilon>0} \lambda^{J, \varepsilon}\left(\bigcup_{i=1}^{\infty} A_{i}\right)=\sup _{J \in \hat{T}, \varepsilon>0} \sup _{n \in \mathbb{N}} \lambda^{J, \varepsilon}\left(\bigcup_{i=1}^{n} A_{i}\right) \\
& =\sup _{n \in \mathbb{N}} \sup _{J \in \hat{T}, \varepsilon>0} \lambda^{J, \varepsilon}\left(\bigcup_{i=1}^{n} A_{i}\right)=\sup _{n \in \mathbb{N}} \sup _{J \in \hat{T}, \varepsilon>0} \sum_{i=1}^{n} \lambda^{J, \varepsilon}\left(A_{i}\right) \\
& =\sup _{n \in \mathbb{N}} \sum_{i=1}^{n} \sup _{J \in \hat{T}, \varepsilon>0}^{\infty} \lambda^{J, \varepsilon}\left(A_{i}\right)=\sum_{i=1}^{\infty} v\left(A_{i}\right),
\end{aligned}
$$

where the fifth equality uses (6.3). Now we will show that measure $v$ satisfies (2.7). Let $I \in \hat{T}$ and $B \in \mathscr{B}\left(\mathbb{R}^{I}\right)$. We have

$$
\begin{aligned}
v\left(\pi_{I}^{-1}\left(B \backslash 0_{I}\right)\right) & \geq \sup _{\varepsilon>0} \lambda^{I, \varepsilon}\left(\pi_{I}^{-1}\left(B \backslash 0_{I}\right)\right) \\
& =\sup _{\varepsilon>0} v_{I}\left(B \cap\left\{y \in \mathbb{R}^{I}: \max _{t \in I}\left|y_{t}\right|>\varepsilon\right\}\right)=v_{I}(B),
\end{aligned}
$$


and, conversely,

$$
\nu_{I}(B)=\nu_{I}\left(B \backslash 0_{I}\right) \geq \lambda_{I}^{J, \varepsilon}\left(B \backslash 0_{I}\right)=\lambda^{J, \varepsilon}\left(\pi_{I}^{-1}\left(B \backslash 0_{I}\right)\right)
$$

for every $J \in \hat{T}$. Taking supremum over $(J, \varepsilon)$ shows $(2.7)$, which completes Step 2.

Step 3. $v$ is a Lévy measure.

We need to show (L2). In view of (2.2), it is enough to show that

$$
v(A)=\sup _{J \in \hat{T}} v\left(A \backslash \pi_{J}^{-1}\left(0_{J}\right)\right) \quad \text { for every } A \in \mathscr{B}^{T} .
$$

First, we will show that for any $J \in \hat{T}$ and $\varepsilon>0$

$$
\sup _{H \in \hat{T}} \lambda^{J, \varepsilon}\left(A \backslash \pi_{H}^{-1}\left(0_{H}\right)\right)=\lambda^{J, \varepsilon}(A) \quad \text { for every } A \in \mathscr{B}^{T} .
$$

By an argument similar to the proof of countable additivity of $v$ in the previous step, we infer that $A \mapsto \sup _{H \in \hat{T}} \lambda^{J, \varepsilon}\left(A \backslash \pi_{H}^{-1}\left(0_{H}\right)\right)$ is a finite measure on $\mathscr{B}^{T}$. Therefore, it is enough to show the equality in (6.5) on the algebra of cylinders. Since " $\leq$ " is obvious, we will prove the opposite inequality. Let $A=\pi_{I}^{-1}(B)$, where $I \in \hat{T}$ and $B \in \mathscr{B}^{I}$, and let $K=I \cup J$. We have

$$
\begin{aligned}
\sup _{H \in \hat{T}} \lambda^{J, \varepsilon}\left(A \backslash \pi_{H}^{-1}\left(0_{H}\right)\right) & \geq \lambda^{J, \varepsilon}\left(A \backslash \pi_{K}^{-1}\left(0_{K}\right)\right)=\lambda^{J, \varepsilon}\left(\pi_{K}^{-1}\left(\pi_{K I}^{-1}(B) \backslash 0_{K}\right)\right) \\
& =\lambda_{K}^{J, \varepsilon}\left(\pi_{K I}^{-1}(B) \backslash 0_{K}\right)=\lambda_{K}^{J, \varepsilon}\left(\pi_{K I}^{-1}(B)\right) \\
& =\lambda^{J, \varepsilon}\left(\pi_{K}^{-1}\left(\pi_{K I}^{-1}(B)\right)\right)=\lambda^{J, \varepsilon}(A) .
\end{aligned}
$$

The second and the fifth equations use (6.2), and the third one follows from the definition of $\lambda_{K}^{J, \varepsilon}$ and that $0_{K} \in\left\{y \in \mathbb{R}^{K}: \max _{t \in J}\left|y_{t}\right| \leq \varepsilon\right\}$. This proves (6.5). Taking supremum over $(J, \varepsilon)$ in (6.5) yields (6.4).

Step 4. $v$ is the smallest measure satisfying (2.7), so is unique.

Suppose that $\rho$ also satisfies (2.7). Let $A=\pi_{I}^{-1}(B)$, where $I \in \hat{T}$ and $B \in \mathscr{B}^{I}$. Using (L2) and Lemma 2.3, we get

$$
\begin{aligned}
v(A) & =v_{*}\left(A \backslash 0_{T}\right)=\sup _{J \in \hat{T}} v\left(A \backslash \pi_{J}^{-1}\left(0_{J}\right)\right)=\sup _{J \in \hat{T}, J \supset I} v\left(\pi_{J}^{-1}\left(\pi_{I J}^{-1}(B) \backslash 0_{J}\right)\right) \\
& =\sup _{J \in \hat{T}, J \supset I} \rho\left(\pi_{J}^{-1}\left(\pi_{I J}^{-1}(B) \backslash 0_{J}\right)\right) \\
& =\sup _{J \in \hat{T}} \rho\left(A \backslash \pi_{J}^{-1}\left(0_{J}\right)\right)=\rho_{*}\left(A \backslash 0_{T}\right)=\rho^{0}(A) .
\end{aligned}
$$

Thus $v=\rho^{0} \leq \rho$ on the algebra of cylinders. By the monotone class argument, the same relation holds on $\mathscr{B}^{T}$. The proof is complete. 
Proof of Proposition 2.10. The integral in (2.11) is well defined by (L1). Given $J \in \hat{T}$ and $a \in \mathbb{R}^{J}$, let $f(x):=\sum_{t \in J} a_{t} x(t)$. Then

$$
\begin{aligned}
\sum_{t \in J} a_{t}\left(\tilde{Y}_{t}-b(t)\right) & =\sum_{t \in J} a_{t} I_{N}(x(t)) \\
& =I_{N}(f)+\int_{\mathbb{R}^{T}} \sum_{t \in J} a_{t} x(t)[\chi(f(x))-\chi(x(t))] v(d x) .
\end{aligned}
$$

Using (2.10), we get

$$
\begin{aligned}
\log \mathbb{E} \exp \left(i \sum_{t \in J} a_{t}\left(\tilde{Y}_{t}-b(t)\right)\right)= & \int_{\mathbb{R}^{T}}\left(e^{i f(x)}-1-i f(x) \chi(f(x))\right) v(d x) \\
& +i \int_{\mathbb{R}^{T}} \sum_{t \in J} a_{t} x(t)[\chi(f(x))-\chi(x(t))] v(d x) \\
= & \int_{\mathbb{R}^{T}}\left(e^{i f(x)}-1-i \sum_{t \in J} a_{t} x(t) \chi(x(t))\right) v(d x) \\
= & \int_{\mathbb{R}^{T}}\left(e^{i\left\langle a, x_{J}\right\rangle}-1-i\left\langle a, \llbracket x_{J} \rrbracket\right\rangle\right) v(d x),
\end{aligned}
$$

which gives (2.6). Therefore, $\widetilde{Y}$ is a version of $Y$.

Proof of THEOREM 2.12. (i) $\Rightarrow$ (ii). There is a set $A \in \mathscr{B}^{T}$ such that $v(A)<$ $\infty$ and $0_{T} \in A$. Then by (2.2)

$$
v(A)=v_{*}\left(A \backslash 0_{T}\right)=\sup _{J \in \hat{T}} v\left(A \backslash \pi_{J}^{-1}\left(0_{J}\right)\right)=\sup _{J \in \hat{T}}\left(v(A)-v\left(A \cap \pi_{J}^{-1}\left(0_{J}\right)\right)\right) .
$$

Hence $\inf _{J \in \hat{T}} v\left(A \cap \pi_{J}^{-1}\left(0_{J}\right)\right)=0$ and $0_{T} \in A \cap \pi_{J}^{-1}\left(0_{J}\right)$, which gives (ii).

(ii) $\Rightarrow$ (iii). Let $A_{n} \in \mathscr{B}^{T}$ be such that $0_{T} \in A_{n}$ and $v\left(A_{n}\right)<n^{-1}$. There exist $T_{n} \in \hat{T}_{c}$ and $B_{n} \in \mathscr{B}^{T_{n}}$, with $0_{T_{n}} \in B_{n}$, such that $A_{n}=\left\{x: x_{T_{n}} \in B_{n}\right\}$. Let $T_{0}=$ $\cup_{n \geq 1} T_{n}$ and $A_{0}=\left\{x: x_{T_{0}}=0_{T_{0}}\right\}$. Since $A_{0} \subset A_{n}$ for every $n \geq 1$, we get $v\left(A_{0}\right)=$ 0 , which proves (iii).

(iii) $\Rightarrow$ (i). Let $A_{0}:=\left\{x: x_{T_{0}}=0\right\}$. By the assumption $v\left(A_{0}\right)=0$, enumerate $T_{0}$ as $T_{0}=\left\{t_{k}: k \in \mathbb{N}\right\}$. For every $k, n \in \mathbb{N}$ set

$$
\begin{aligned}
A_{k, n} & :=\left\{x:\left|x\left(t_{k}\right)\right| \geq n^{-1}\right\}, \\
v\left(A_{k, n}\right) & =v\left\{x:\left|x\left(t_{k}\right)\right| \wedge 1 \geq n^{-1}\right\} \leq n^{2} \int_{\mathbb{R}^{T}}\left|x\left(t_{k}\right)\right|^{2} \wedge 1 v(d x)<\infty .
\end{aligned}
$$

Since $A_{0} \cup \bigcup_{k, n \in \mathbb{N}} A_{k, n}=\mathbb{R}^{T}$, (i) holds.

Proof of TheOREM 2.14. Assume that $v$ is not $\sigma$-finite. Let $\tilde{Y}$ be the canonical spectral representation of $Y$. That is,

$$
\tilde{Y}_{t}=\int_{\{x(t) \neq 0\}} x(t)[N(d x)-\chi(x(t)) v(d x)]+b(t), \quad t \in T,
$$


where $N$ is a Poisson random measure on $\mathbb{R}^{T}$ with intensity $v$. Let $T_{0} \in \hat{T}_{c}$. By Corollary 2.13, there is $t_{1} \notin T_{0}$ such that $\nu(A)>0$, where

$$
A=\left\{x \in \mathbb{R}^{T}: x_{T_{0}}=0, x\left(t_{1}\right) \neq 0\right\} .
$$

Define

$$
\begin{aligned}
& \eta=\int_{A} x\left(t_{1}\right)\left[N(d x)-\chi\left(x\left(t_{1}\right)\right) v(d x)\right] \text { and } \\
& \xi=\int_{B} x\left(t_{1}\right)\left[N(d x)-\chi\left(x\left(t_{1}\right)\right) v(d x)\right]+b\left(t_{1}\right),
\end{aligned}
$$

where $B=\left\{x \in \mathbb{R}^{T}: x_{T_{0}} \neq 0, x\left(t_{1}\right) \neq 0\right\}$. Clearly, conditions (a)-(c) hold.

Conversely, suppose (a)-(c) hold for some version $\tilde{Y}$ of $Y$. Let $T_{1}=T_{0} \cup\left\{t_{1}\right\}$ and $U=\left(U_{t}: t \in T_{1}\right)$ be given by $U_{t}=\widetilde{Y}_{t}$ when $t \in T_{0}$ and $U_{t_{1}}=\xi$; define also $V=\left(V_{t}: t \in T_{1}\right)$ by $V_{T_{0}}=0$ and $V_{t_{1}}=\eta$. Then $\widetilde{Y}_{T_{1}}=U+V$ and processes $U$ and $V$ are independent. Let $v_{\widetilde{Y}_{T_{1}}}, v_{U}$ and $v_{V}$ be Lévy measures on $\left(\mathbb{R}^{T_{1}, \mathscr{B}^{T_{1}}}\right)$ of $\widetilde{Y}_{T_{1}}$, $U$ and $V$, respectively. We have $v_{Y_{T_{1}}}=v_{U}+v_{V}$. Moreover, $v_{V}$ is concentrated on the $t_{1}$-axis, that is, $v_{V}=\delta_{0_{T_{0}}} \otimes v_{\eta}$ with $v_{\eta}$ being a Lévy measure of $\eta$. Hence

$$
\begin{aligned}
v\{x & \left.\in \mathbb{R}^{T}: x_{T_{0}}=0, x\left(t_{1}\right) \neq 0\right\} \\
& =v\left(\pi_{T_{1}}^{-1}\left(0_{T_{0}} \times(\mathbb{R} \backslash\{0\})\right)\right) \\
& =v_{\widetilde{Y}_{T_{1}}}\left(0_{T_{0}} \times(\mathbb{R} \backslash\{0\})\right) \geq v_{V}\left(0_{T_{0}} \times(\mathbb{R} \backslash\{0\})\right)=v_{\eta}(\mathbb{R})>0
\end{aligned}
$$

since $\eta$ is nondegenerate. By Corollary 2.13, $v$ is not $\sigma$-finite, which completes the proof.

Proof of Lemma 2.21. Let $V$ be as in Definition 2.20. Let $f: S \mapsto(0, \infty)$ be a measurable function such that $\int_{S} f(s) n(d s)<\infty$ and let $n_{1}(d s):=f(s) n(d s)$ be a finite measure on $S$. Put

$$
\alpha=\inf _{J \in \hat{T}} n_{1}\left\{s \in S: V_{J}(s)=0\right\} .
$$

Then $\alpha \in[0, \infty)$ and there is $T_{0} \in \hat{T}_{c}$ such that $\alpha=n_{1}\left\{s \in S: V_{T_{0}}(s)=0\right\}$.

Let $B \in \mathscr{B}_{0}^{I}, I \in \hat{T}$, and let $T_{1}=T_{0} \cup I$. Since $\left\{V_{I} \in B\right\} \subset\left\{V_{T_{1}} \neq 0\right\}$ and by the extremity of $T_{0}, n_{1}\left\{V_{T_{0}}=0, V_{T_{1}} \neq 0\right\}=0$ so that $n\left\{V_{T_{0}}=0, V_{T_{1}} \neq 0\right\}=0$, we get

$$
\begin{aligned}
v_{I}(B) & =n\left\{V_{I} \in B\right\}=n\left\{V_{I} \in B, V_{T_{1}} \neq 0\right\} \\
& =n\left\{V_{I} \in B, V_{T_{0}}=0, V_{T_{1}} \neq 0\right\}+n\left\{V_{I} \in B, V_{T_{0}} \neq 0, V_{T_{1}} \neq 0\right\} \\
& =n\left\{V_{I} \in B, V_{T_{0}} \neq 0\right\} .
\end{aligned}
$$

Therefore, $V$ restricted to $S_{0}=\left\{s \in S: V_{T_{0}}(s) \neq 0\right\}$ is a representation of $v$ and is exact because it satisfies (2.1) [which implies (L2)]. 
Proof of Theorem 3.2. Consider on some probability space $\left(\Omega^{\prime}, \mathscr{F}^{\prime}, \mathbb{P}^{\prime}\right)$ mutually independent centered Gaussian process $G^{\prime}$ over $T$ with covariance $\Sigma$ and a Poisson random measure $N^{\prime}$ on $(S, \mathscr{S})$ with intensity $n$. By Proposition 3.1 (with $G=G^{\prime}$ and $N=N^{\prime}$ ), we have $X \stackrel{d}{=} X^{\prime}=G^{\prime}+Y^{\prime}$, where $Y^{\prime}$ is the Poissonian part in (3.1). Now we restrict the index set to $T_{0}$ and write (3.1) as

$$
X_{T_{0}} \stackrel{d}{=} G_{T_{0}}^{\prime}+Y_{T_{0}}^{\prime}=f\left(G_{T_{0}}^{\prime}, N_{\mathscr{S}_{0}}^{\prime}\right),
$$

where $N_{\mathscr{S}_{0}}^{\prime}$ is the restriction of $N^{\prime}$ to a countable algebra $\mathscr{S}_{0}$ that generates $\mathscr{S}$ modulo $n$. Indeed, since the stochastic integral with respect to $N^{\prime}$ is a limit of integrals of simple functions and $N^{\prime}$ on $\mathscr{S}$ can be approximated by $N^{\prime}$ on $\mathscr{S}_{0}$, the right-hand side of (3.1) (restricted to $T_{0}$ ) can be represented as a Borel function $f$ of a random element $\left(G_{T_{0}}^{\prime}, N_{\mathscr{S}_{0}}^{\prime}\right)$ taking values in a Polish space $\mathbb{R}^{T_{0}} \times[0, \infty]^{\mathscr{S}_{0}}$. By [11], Corollary 6.11, there exists a random element $\left(\left(G_{t}\right)_{t \in T_{0}},(N(A))_{A \in \mathscr{S}_{0}}\right)$ on the original probability space of $X$ such that

$$
X_{T_{0}}=f\left(\left(G_{t}\right)_{t \in T_{0}},(N(A))_{A \in \mathscr{S}_{0}}\right) \quad \text { a.s. }
$$

and $\left(\left(G_{t}\right)_{t \in T_{0}},(N(A))_{A \in \mathscr{S}_{0}}\right) \stackrel{d}{=}\left(G_{T_{0}}^{\prime}, N_{\mathscr{S}_{0}}^{\prime}\right)$. Since $(N(A))_{A \in \mathscr{S}_{0}}$ is a Poisson random measure on the algebra $\mathscr{S}_{0}$, independent of $\left(G_{t}\right)_{t \in T_{0}}$, it extends uniquely to a Poisson random measure $N$ on $(S, \mathscr{S}, n)$, which is also independent of $\left(G_{t}\right)_{t \in T_{0}}$. Therefore, (6.6) establishes (3.2) for $t \in T_{0}$ (see [18], Theorem 5.2, for more details).

Since $X$ is separable in probability, for every $t \in T$ there exists $\tau_{n} \in T_{0}$ such that $X_{\tau_{n}} \stackrel{P}{\rightarrow} X_{t}$. By a symmetrization inequality, $G_{t}:=\lim _{n \rightarrow \infty} G_{\tau_{n}}$ exists in probability and $G=\left\{G_{t}\right\}_{t \in T}$ is independent of $N$. Having $G$ and $N$ constructed, we use Proposition 3.1 again to state that

$$
X_{t}^{\prime \prime}:=G_{t}+\int_{S} V_{t}\left(d N-\chi\left(V_{t}\right) d n\right)+b(t), \quad t \in T
$$

is a version of $X$. Since $X_{t}=X_{t}^{\prime \prime}$ a.s. for each $t \in T_{0}$, and $T_{0}$ is a common separant for both $X$ and $X^{\prime \prime}$, we get $X_{t}=X_{t}^{\prime \prime}$ a.s. for all $t \in T$. This establishes (3.2) and completes the proof.

Proof OF THEOREM 3.4. Let $f: S \mapsto(0,1]$ be a measurable function such that $\int_{S} f(s) n(d s)<\infty$. Consider a finite measure $n_{0}(d s):=f(s) n(d s)$ on $S$. Let $\xi^{k}=\left(\xi_{t}^{k}\right)_{t \in T}$ be an i.i.d. sequence of processes over $T$ with the common distribution $\theta^{-1} n_{0} \circ V^{-1}$, where $\theta=n_{0}(S)$, so that

$$
n_{0} \circ V^{-1}(B)=\theta \mathbb{P}\left(\xi^{k} \in B\right), \quad B \in \mathscr{B}^{T} .
$$

Let $Y_{t}:=\sum_{k=1}^{\eta} \xi_{t}^{k}$ be a compound Poisson process, where $\eta$ is a Poisson random variable with mean $\theta$ and independent of $\left\{\xi^{k}\right\}$. Put $v_{0}:=n_{0} \circ V^{-1}$ and notice that for every $B \in \mathscr{B}^{T}$

$$
v_{0}(B)=\int_{S} \mathbf{1}_{B}(V(s)) f(s) n(d x) \leq \int_{S} \mathbf{1}_{B}(V(s)) n(d x)=v(B) .
$$


Therefore, $v_{0}$ is equivalent to $v$ and $v_{0} \leq v$. Since $v$ is $\sigma$-finite, $v$ satisfies condition (2.1), so does $v_{0}$. Therefore, $v_{0}$ is a Lévy measure of $Y$ (see Example 2.26). Suppose that the process $X$ has the generating triplet $(\Sigma, v, b)$. Let $Z=\left(Z_{t}\right)_{t \in T}$ be an infinitely divisible process independent of $\left\{\eta, \xi_{t}^{k}: t \in T, k \in \mathbb{N}\right\}$ and with the generating triplet $\left(\Sigma, v-v_{0}, c\right)$, where $c$ is a shift function such that

$$
X \stackrel{d}{=} Y+Z .
$$

By Lemma 6.1 given below, there exists $\tilde{X}$ with all paths in $U$ such that $\tilde{X}_{t}=$ $Y_{t}+Z_{t}$ a.s. for each $t \in T$. We will now check that $Z$ satisfies (6.7). For every $A \subset \mathbb{R}^{T} \backslash U, A \in \mathscr{B}^{T}$ we get

$$
\mathbb{P}(Z \in A)=e^{\theta} \mathbb{P}(Z \in A, \eta=0)=e^{\theta} \mathbb{P}(Y+Z \in A, \eta=0) \leq e^{\theta} \mathbb{P}(\tilde{X} \in A)=0 .
$$

Thus, there exists $\widetilde{Z}$ with all paths in $U$ such that $\widetilde{Z}_{t}=Z_{t}$ a.s. for each $t \in T$. Hence, by our assumption on $U, \widetilde{Y}:=\widetilde{X}-\widetilde{Z}$ is a modification of $Y$ with all paths in $U$. Consider the representation $V$ as a stochastic process under the probability measure $\theta^{-1} n_{0}$, so we have $V \stackrel{d}{=} \xi^{1}$. For any set $A$ as above, we have

$$
\begin{aligned}
\theta^{-1} n_{0}(V \in A) & =\mathbb{P}\left(\xi^{1} \in A\right)=\theta^{-1} e^{\theta} \mathbb{P}\left(\xi^{1} \in A, \eta=1\right) \\
& =\theta^{-1} e^{\theta} \mathbb{P}(\tilde{Y} \in A, \eta=1)=0 .
\end{aligned}
$$

By Lemma 6.1, there exists a process $\widetilde{V}=\left(\widetilde{V}_{t}\right)_{t \in T}$ with all paths in $U$ such that

$$
\theta^{-1} n_{0}\left\{s: \widetilde{V}_{t}(s) \neq V_{t}(s)\right\}=0 \quad \text { for all } t \in T .
$$

Since the measures $n$ and $\theta^{-1} n_{0}$ are equivalent, this proof is complete.

Lemma 6.1. Let $X=\left(X_{t}\right)_{t \in T}$ be a stochastic process and let $U \subset \mathbb{R}^{T}$. Assume that $(U, \mathscr{U})$ is a Borel space for the $\sigma$-algebra $\mathscr{U}=\mathscr{B}^{T} \cap U$. Then there exists a process $\widetilde{X}$ with all paths in $U$ such that $\widetilde{X}_{t}=X_{t}$ a.s. for every $t \in T$ if and only if

$$
\mathbb{P}(X \in A)=0 \quad \text { for all } A \subset \mathbb{R}^{T} \backslash U, A \in \mathscr{B}^{T} .
$$

PROOF OF Lemma 6.1. The necessity of (6.7) is obvious, so we will prove its sufficiency. Define for every $B \in \mathscr{U}$

$$
\mu(B):=\mathbb{P}(X \in A) \quad \text { when } A \cap U=B, A \in \mathscr{B}^{T} .
$$

It is routine to check that under (6.7) $\mu$ is a well-defined probability measure on $(U, \mathscr{U})$. Let $Y_{t}(u):=u(t), t \in T, u \in U$. Then $Y=\left(Y_{t}\right)_{t \in T}$ is a stochastic process defined on $(U, \mathscr{U}, \mu)$ with paths in $U$. For every $A \in \mathscr{B}^{T}$,

$$
\mu(Y \in A)=\mu(Y \in A \cap U)=\mathbb{P}(X \in A),
$$

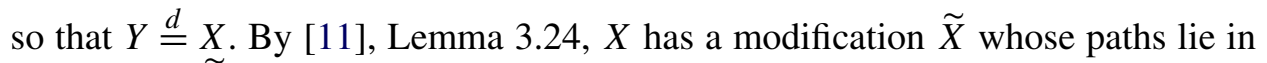
$U$ such that $\widetilde{X}_{t}=X_{t}$ a.s. for each $t \in T$. 
As we have mentioned in Section 4, Theorem 4.1 is a consequence of Theorem 4.3, which itself is deduced from Theorem 4.4. Therefore, we begin with Theorem 4.4.

Proof of TheOrem 4.4. Notice that $\mathbb{E} N(q)=\int_{S} q d n=1$. Let $Y=X-G$ be the Poissonian part of $X$. It is enough to prove (4.7) for $F$ of the form $F(x)=\exp \left\{i \sum_{j=1}^{n} a_{j} x\left(t_{j}\right)\right\}$, where $a_{1}, \ldots, a_{n} \in \mathbb{R}, t_{1}, \ldots, t_{n} \in T, n \geq 1$. We have $F(X)=F(G) F(Y)$, where

$$
F(Y)=\exp \left\{i \sum_{j=1}^{n} a_{j}\left[\int_{S} V_{t_{j}}\left(d N-\chi\left(V_{t_{j}}\right) d n\right)+b\left(t_{j}\right)\right]\right\}=H(N)
$$

Here, $N=(N(A))_{A \in \mathscr{S}}$ is viewed as a stochastic process and $H: \mathbb{R}^{\mathscr{S}} \mapsto \mathbb{R}$ is a measurable functional. Using the independence and Mecke-Palm formula, we get

$$
\begin{aligned}
\mathbb{E}[F(X) N(q)] & =\mathbb{E}[F(G) H(N) N(q)]=\mathbb{E}[F(G)] \mathbb{E}[H(N) N(q)] \\
& =\mathbb{E}[F(G)] \mathbb{E} \int_{S} q(s) H(N) N(d s) \\
& =\mathbb{E}[F(G)] \int_{S} \mathbb{E}\left[q(s) H\left(N+\delta_{s}\right)\right] n(d s) \\
& =\mathbb{E}[F(G)] \int_{S} \mathbb{E}[q(s) F(V(s)) H(N)] n(d s) \\
& =\mathbb{E}[F(G)] \mathbb{E}[H(N)] \int_{S} F(V(s)) q(s) n(d s) \\
& =\mathbb{E}[F(X)] \int_{S} F(V(s)) q(s) n(d s) \\
& =\int_{S} \mathbb{E} F(X+V(s)) q(s) n(d s) .
\end{aligned}
$$

This establishes (4.7).

To prove (4.8), notice that $X$ and $N(q)$ are jointly infinitely divisible. Let $\theta$ be an isolated point of $T$ and put $T_{\theta}=T \cup\{\theta\}$. Consider an infinitely divisible process $\bar{X}=\left(\bar{X}_{t}\right)_{t \in T_{\theta}}$ given by

$$
\bar{X}_{t}= \begin{cases}X_{t} & t \in T \\ N(q) & t=\theta\end{cases}
$$

The Lévy measure of $\bar{X}$ has a representation $\bar{V}$ on $(S, \mathscr{S}, n)$ of the form

$$
\bar{V}_{t}= \begin{cases}V_{t} & t \in T \\ q & t=\theta\end{cases}
$$


Let $H: \mathbb{R}^{T_{\theta}} \mapsto \mathbb{R}$ be given by $H(x)=F\left(x_{T}\right) \frac{1}{x_{\theta}} \mathbf{1}\left(x_{\theta}>0\right), x \in T_{\theta}$, where $F$ is as above. Applying (4.7), we get

$$
\begin{aligned}
\mathbb{E}[F & \left.\left(\left(X_{t}\right)_{t \in T}\right) ; N(q)>0\right] \\
& =\mathbb{E}\left[H\left(\left(\bar{X}_{t}\right)_{t \in T_{\theta}}\right) ; N(q)\right] \\
& =\int_{S} \mathbb{E} H\left(\left(\bar{X}_{t}+\bar{V}_{t}(s)\right)_{t \in T_{\theta}}\right) q(s) n(d s) \\
& =\int_{S} \mathbb{E} F\left(\left(X_{t}+V_{t}(s)\right)_{t \in T}\right) \frac{\mathbf{1}(N(q)+q(s)>0)}{N(q)+q(s)} q(s) n(d s) \\
& =\int_{S} \mathbb{E}\left[F\left(\left(X_{t}+V_{t}(s)\right)_{t \in T}\right) ;(N(q)+q(s))^{-1}\right] q(s) n(d s),
\end{aligned}
$$

which shows (4.8).

The last formula (4.9) follows from the previous (4.8) since

$$
\begin{aligned}
\mathbb{P}(N(q)>0) & =1-\lim _{u \rightarrow \infty} \mathbb{E} e^{-u N(q)}=1-\lim _{u \rightarrow \infty} \exp \left(\int_{S}\left[e^{-u q(s)}-1\right] n(d s)\right) \\
& =1-\exp (-n\{q(s)>0\}) .
\end{aligned}
$$

The proof is complete.

Proof of TheOrem 4.3. First, we will show that part (a) follows from part (b) of this theorem. Indeed, suppose $\mathscr{L}(Z) \ll v$, so that $\mathscr{L}(Z) \ll v+\delta_{0_{T}}$. Let $q=\frac{d \mathscr{L}(Z)}{d\left(v+\delta_{0_{T}}\right)}$. Since $v$ is $\sigma$-finite, $v\left\{x: x_{T_{0}}=0\right\}=0$ for some $T_{0} \in \hat{T}_{c}$, so that $\mathbb{P}\left(Z_{T_{0}}=0\right)=0$. We have

$$
0=\mathbb{P}\left(Z_{T_{0}}=0\right)=\int_{\left\{x: x_{T_{0}}=0\right\}} q(x) v(d x)+q\left(0_{T}\right) \delta_{0_{T}}\left(\left\{x: x_{T_{0}}=0\right\}\right)=q\left(0_{T}\right) .
$$

Hence, $q\left(0_{T}\right)=0$, in which case (4.5) becomes (4.3) and (4.6) becomes (4.4). Therefore, we only need to prove (b).

Let $S=\mathbb{R}^{T}$ and $\mathscr{S}=\mathscr{B}^{T}$. Consider $N_{1}=N+\eta \delta_{0_{T}}$, where $\eta$ is a Poisson random variable with mean 1 independent of $N$ and $G . N_{1}$ is a Poisson random measure on $(S, \mathscr{S})$ with intensity $n_{1}=v+\delta_{0_{T}}$. Notice that (4.2) still holds after replacing $N$ by $N_{1}$ and $v$ by $n_{1}$. Therefore, $V_{t}(x)=x(t)$ is a representation of $v$ on $\left(S, \mathscr{S}, n_{1}\right)$. Using Theorem 4.4 , we get

$$
\begin{aligned}
\mathbb{E} F\left(\left(X_{t}+Z_{t}\right)_{t \in T}\right) & =\int_{S} \mathbb{E} F\left(\left(X_{t}+x(t)\right)_{t \in T}\right) q(x) n(d x) \\
& =\mathbb{E}\left[F\left(\left(X_{t}\right)_{t \in T}\right) ; N_{1}(q)\right]=\mathbb{E}\left[F\left(\left(X_{t}\right)_{t \in T}\right) ; N(q)+\eta q\left(0_{T}\right)\right] \\
& =\mathbb{E}\left[F\left(\left(X_{t}\right)_{t \in T}\right) ; N(q)\right]+\mathbb{E}\left[F\left(\left(X_{t}\right)_{t \in T}\right)\right] q\left(0_{T}\right),
\end{aligned}
$$

by the independence of $N, G$ and $\eta$, and $\mathbb{E} \eta=1$. This proves (4.5). 
Since $\mathscr{L}(Z) \ll n_{r}:=v+r \delta_{0_{T}}$ for every $r>0$, we may consider $q_{r}:=\frac{d \mathscr{L}(Z)}{d n_{r}}$, so that $q_{1}=q$. Because $v$ and $\delta_{0_{T}}$ are singular, $q_{r}\left(0_{T}\right)=r^{-1} q\left(0_{T}\right)$ and $q_{r}=q$ $v$-a.e. Therefore, we can take $q_{r}=q \mathbf{1}_{U^{c}}+r^{-1} q\left(0_{T}\right) \mathbf{1}_{U}$ as a version of $q_{r}$, where $U$ is any set such that $0_{T} \in U \in \mathscr{B}^{T}$ and $v(U)=0$.

Let $\eta_{r}$ be a Poisson random variable with mean $r$, independent of $N$ and $G$, and let $N_{r}=N+\eta_{r} \delta_{0_{T}}$. By the same argument as above, with $n_{1}$ replaced by $n_{r}$ and $N_{1}$ replaced by $N_{r}$, we use (4.8) of Theorem 4.4 to get

$$
\begin{aligned}
& \mathbb{E}\left[F\left(\left(X_{t}\right)_{t \in T}\right) ; N_{r}\left(q_{r}\right)>0\right] \\
& \quad=\mathbb{E}\left[F\left(\left(X_{t}+Z_{t}\right)_{t \in T}\right)\left(N_{r}\left(q_{r}\right)+q_{r}(Z)\right)^{-1}\right],
\end{aligned}
$$

which can written as

$$
\begin{aligned}
& \mathbb{E}\left[F\left(\left(X_{t}\right)_{t \in T}\right) ; N(q)+r^{-1} \eta_{r} q\left(0_{T}\right)>0\right] \\
& =\mathbb{E}\left[F ( ( X _ { t } + Z _ { t } ) _ { t \in T } ) \left(N(q)+r^{-1} \eta_{r} q\left(0_{T}\right)+q(Z) \mathbf{1}_{U^{c}}(Z)\right.\right. \\
& \left.\left.\quad+r^{-1} q\left(0_{T}\right) \mathbf{1}_{U}(Z)\right)^{-1}\right]
\end{aligned}
$$

Letting $r \rightarrow \infty$, and using that $r^{-1} \eta_{r} \stackrel{P}{\rightarrow} 1$, we get

$$
\begin{aligned}
\mathbb{E}\left[F\left(\left(X_{t}\right)_{t \in T}\right)\right] & =\mathbb{E}\left[F\left(\left(X_{t}\right)_{t \in T}\right) ; N(q)+q\left(0_{T}\right)>0\right] \\
& =\mathbb{E}\left[F\left(\left(X_{t}+Z_{t}\right)_{t \in T}\right)\left(N(q)+q\left(0_{T}\right)+q(Z) \mathbf{1}_{U^{c}}(Z)\right)^{-1}\right] \\
& =\mathbb{E}\left[F\left(\left(X_{t}+Z_{t}\right)_{t \in T}\right)\left(N(q)+q(Z)+q\left(0_{T}\right) \mathbf{1}_{U^{c}}(Z)\right)^{-1}\right]
\end{aligned}
$$

because $q(Z) \mathbf{1}_{U}(Z)=q\left(0_{T}\right) \mathbf{1}_{U}(Z)$ a.s.

Finally, $\mathscr{L}(X+Z) \ll \mathscr{L}(X)$ follows from (4.3). Conversely, notice that $N(q)+$ $q\left(0_{T}\right)>0$ a.s. if either $q\left(0_{T}\right)>0$ or, by (6.8), $v\{x: q(x)>0\}=\infty$. In these cases, (4.4) gives $\mathscr{L}(X) \ll \mathscr{L}(X+Z)$. The proof is complete.

Proof OF THEOREM 4.1. Without loss of generality, we may assume that $X=G+Y$, where $G$ is s centered Gaussian process, $Y$ is the Poissonian part of $X$ given by a canonical spectral representation (4.2) relative to a Poisson random measure $N$, where $G, N$ and $Z$ are independent. By Theorem 4.3,

$$
\mathbb{E} F\left(\left(X_{t}+Z_{t}\right)_{t \in T}\right)=\mathbb{E}\left[F\left(\left(X_{t}\right)_{t \in T}\right) ; N(q)\right],
$$

which implies

$$
\mathscr{L}(X+Z) \ll \mathscr{L}(X)
$$

Therefore, $\mathbb{E} F\left(\left(X_{t}+Z_{t}\right)_{t \in T}\right)=\mathbb{E}\left[F\left(\left(X_{t}\right)_{t \in T}\right) ; g(X)\right]$, where $g(x)=$ $\frac{d \mathscr{L}(X+Z)}{d \mathscr{L}(X)}(x), x \in \mathbb{R}^{T}$. 
PROOF OF LEMMA 4.6. We follow, with some necessary modifications, arguments from [7], Lemma 3.1. Assume (i). Then the Laplace transform of $Y$ is of the form

$$
\begin{aligned}
\phi\left(\alpha_{1}, \ldots, \alpha_{n}\right) & =\mathbb{E} \exp \left\{-\sum_{i=1}^{n} \alpha_{i} Y_{i}\right\} \\
& =\exp \left\{-\sum_{i=1}^{n} \alpha_{i} c_{i}-\int_{\mathbb{R}_{+}^{n}}\left(1-e^{-\sum_{i=1}^{n} \alpha_{i} y_{i}}\right) v(d y)\right\}
\end{aligned}
$$

where $\alpha_{i}, c_{i} \geq 0$. We have

$$
\frac{\partial}{\partial \alpha_{k}} \phi\left(\alpha_{1}, \ldots, \alpha_{n}\right)=-\phi\left(\alpha_{1}, \ldots, \alpha_{n}\right)\left[c_{k}+\int_{\mathbb{R}_{+}^{n}} e^{-\sum_{i=1}^{n} \alpha_{i} y_{i}} y_{k} v(d y)\right] .
$$

Hence, $\theta_{k}=\mathbb{E}\left(Y_{k}\right)=c_{k}+\int_{\mathbb{R}_{+}^{n}} y_{k} v(d y)$. Let $Z^{k}$ be a vector in $\mathbb{R}_{+}^{n}$ independent of $Y$ whose distribution is given by

$$
\mathscr{L}\left(Z^{k}\right)(d y)=\frac{c_{k}}{\theta_{k}} \delta_{(0, \ldots, 0)}(d y)+\frac{y_{k}}{\theta_{k}} v(d y) .
$$

Using (6.9), we obtain

$$
\mathbb{E}\left[\exp \left\{-\sum_{i=1}^{n} \alpha_{i} Y_{i}\right\} ; \theta_{k}^{-1} Y_{k}\right]=\mathbb{E}\left[\exp \left\{-\sum_{i=1}^{n} \alpha_{i}\left(Y_{i}+Z_{i}^{k}\right)\right\}\right],
$$

which yields (ii).

Assume (ii). We will prove that $Y$ is infinitely divisible with the Lévy measure and drift given by (4.16)-(4.17). To this end, we first show that for any bounded measurable functional $F: \mathbb{R}^{n} \mapsto \mathbb{R}$ and $j, k \leq n$

$$
\theta_{j} \mathbb{E}\left[F\left(Z^{j}\right) Z_{k}^{j}\right]=\theta_{k} \mathbb{E}\left[F\left(Z^{k}\right) Z_{j}^{k}\right] .
$$

It is enough to show (6.10) for $F(y)=\exp \left\{-\sum_{i=1}^{n} \alpha_{i} y_{i}\right\}$, where $\alpha_{i}>0$. Using (4.15) twice and independence, we get

$$
\begin{aligned}
\mathbb{E}\left[F(Y) Y_{j} ; Y_{k}\right] & =\theta_{k} \mathbb{E}\left[F\left(Y+Z^{k}\right)\left(Y_{j}+Z_{j}^{k}\right)\right] \\
& =\theta_{k} \mathbb{E}\left[F\left(Y+Z^{k}\right) ; Y_{j}\right]+\theta_{k} \mathbb{E}\left[F\left(Y+Z^{k}\right) Z_{j}^{k}\right] \\
& =\theta_{k} \mathbb{E}\left[F(Y) ; Y_{j}\right] \mathbb{E}\left[F\left(Z^{k}\right)\right]+\theta_{k} \mathbb{E}\left[F\left(Z^{k}\right) Z_{j}^{k}\right] \mathbb{E}[F(Y)] \\
& =\theta_{j} \theta_{k} \mathbb{E}[F(Y)] \mathbb{E}\left[F\left(Z^{j}\right)\right] \mathbb{E}\left[F\left(Z^{k}\right)\right]+\theta_{k} \mathbb{E}\left[F\left(Z^{k}\right) Z_{j}^{k}\right] \mathbb{E}[F(Y)] .
\end{aligned}
$$

Since interchanging $j$ and $k$ does not change the first term in these equations, equating the final terms after the interchange gives (6.10). Taking $F(y)=\mathbf{1}_{\left\{y_{j}=0\right\}}$ in (6.10) yields

$$
\theta_{j} \mathbb{E}\left[\mathbf{1}_{\left\{Z_{j}^{j}=0\right\}} Z_{k}^{j}\right]=\theta_{k} \mathbb{E}\left[\mathbf{1}_{\left\{Z_{j}^{k}=0\right\}} Z_{j}^{k}\right]=0
$$


This implies that for every $j, k \leq n$,

$$
\left\{Z_{k}^{j}>0\right\} \subset\left\{Z_{j}^{j}>0\right\} \quad \text { a.s. }
$$

Applying (6.10) to $F(y)\left(y_{j} y_{k}\right)^{-1} \mathbf{1}_{\left\{y_{j} y_{k}>0\right\}}$ in the place of $F$, where $F \geq 0$, and taking into account (6.11), we obtain

$$
\theta_{j} \mathbb{E}\left[F\left(Z^{j}\right) \mathbf{1}_{\left\{Z_{k}^{j}>0\right\}}\left(Z_{j}^{j}\right)^{-1}\right]=\theta_{k} \mathbb{E}\left[F\left(Z^{k}\right) \mathbf{1}_{\left\{Z_{j}^{k}>0\right\}}\left(Z_{k}^{k}\right)^{-1}\right] .
$$

Now we are ready to prove (i) together with (4.16)-(4.17). For $n=1$, we get from (4.15)

$$
\mathbb{E}\left[e^{-\alpha Y} Y\right]=\theta \mathbb{E} e^{-\alpha(Y+Z)}=\theta \mathbb{E} e^{-\alpha Y} \mathbb{E} e^{-\alpha Z},
$$

which yields

$$
\frac{d}{d \alpha} \log \mathbb{E}\left[e^{-\alpha Y}\right]=-\theta \mathbb{E} e^{-\alpha Z}
$$

Therefore,

$$
\begin{aligned}
\mathbb{E}\left[e^{-\alpha Y}\right] & =\exp \left\{-\theta \mathbb{E} \int_{0}^{\alpha}\left(\mathbf{1}_{\{Z=0\}}+e^{-s Z} \mathbf{1}_{\{Z>0\}}\right) d s\right\} \\
& =\exp \left\{-\alpha \theta \mathbb{P}(Z=0)-\theta \int_{0}^{\infty}\left(1-e^{-\alpha y}\right) \mathbf{1}\{y>0\} y^{-1} \mathscr{L}(Z)(d y)\right\},
\end{aligned}
$$

which shows our claim.

We proceed by induction. Assuming (ii), suppose that (i) and (4.16)-(4.17) hold for $n-1$. Since $\tilde{Y}:=Y_{\{1, \ldots, n-1\}}$ and $\tilde{Z}^{j}:=Z_{\{1, \ldots, n-1\}}^{j}(j \leq n-1)$ satisfy (ii) for $n-1$ in the place of $n$, by the induction hypothesis $\tilde{Y}$ is infinitely divisible with the Laplace transform

$$
\tilde{\phi}\left(\alpha_{1}, \ldots, \alpha_{n-1}\right)=\exp \left\{-\sum_{i=1}^{n-1} \alpha_{i} \tilde{c}_{i}-\int_{\mathbb{R}_{+}^{n-1}}\left(1-e^{-\sum_{i=1}^{n-1} \alpha_{i} y_{i}}\right) \tilde{v}(d y)\right\},
$$

where $\tilde{c}=\left(\theta_{1} \mathbb{P}\left(Z_{1}^{1}=0\right), \ldots, \theta_{n} \mathbb{P}\left(Z_{n-1}^{n-1}=0\right)\right)$ and

$$
\tilde{v}(d y)=\sum_{k=1}^{n-1} \theta_{k} \mathbf{1}_{\left\{y_{1}=\cdots=y_{k-1}=0, y_{k}>0\right\}} y_{k}^{-1} \mathscr{L}\left(\tilde{Z}^{k}\right)(d y) .
$$

Let $\phi\left(\alpha_{1}, \ldots, \alpha_{n}\right)=\mathbb{E} \exp \left\{-\sum_{i=1}^{n} \alpha_{i} Y_{i}\right\}$. Proceeding as before, we get

$$
\frac{\partial}{\partial \alpha_{n}} \log \phi\left(\alpha_{1}, \ldots, \alpha_{n}\right)=-\theta_{n} \mathbb{E} \exp \left\{-\sum_{i=1}^{n} \alpha_{i} Z_{i}^{n}\right\} \text {. }
$$

Hence,

$$
\begin{aligned}
& \phi\left(\alpha_{1}, \ldots, \alpha_{n}\right) \\
& \quad=\tilde{\phi}\left(\alpha_{1}, \ldots, \alpha_{n-1}\right) \exp \left\{-\theta_{n} \mathbb{E}\left[e^{-\sum_{i=1}^{n-1} \alpha_{i} Z_{i}^{n}} \int_{0}^{\alpha_{n}} e^{-s Z_{n}^{n}} d s\right]\right\} .
\end{aligned}
$$


Notice that $Z_{i}^{n}=0$ a.s. on the set $\left\{Z_{n}^{n}=0\right\}$ by (6.11). Therefore, the exponent of the last term on the right-hand side of (6.13) equals

$$
\begin{aligned}
-\theta_{n} \mathbb{E} & {\left[\alpha_{n} \mathbf{1}_{\left\{Z_{n}^{n}=0\right\}}+e^{-\sum_{i=1}^{n-1} \alpha_{i} Z_{i}^{n}}\left(1-e^{-\alpha_{n} Z_{n}^{n}}\right) \mathbf{1}_{\left\{Z_{n}^{n}>0\right\}}\left(Z_{n}^{n}\right)^{-1}\right] } \\
& =-\alpha_{n} c_{n}-\theta_{n} \sum_{k=1}^{n} \mathbb{E}\left[e^{-\sum_{i=1}^{n-1} \alpha_{i} Z_{i}^{n}}\left(1-e^{-\alpha_{n} Z_{n}^{n}}\right) \mathbf{1}_{\left\{Z_{1}^{n}=\cdots=Z_{k-1}^{n}=0, Z_{k}^{n}>0\right\}}\left(Z_{n}^{n}\right)^{-1}\right],
\end{aligned}
$$

which, after applying (6.12) and noticing that the term $Z_{n}^{k}>0$ can be replaced by $Z_{k}^{k}>0$, gives us

$$
\begin{aligned}
= & -\alpha_{n} c_{n}-\sum_{k=1}^{n} \theta_{k} \mathbb{E}\left[e^{-\sum_{i=1}^{n-1} \alpha_{i} Z_{i}^{k}}\left(1-e^{-\alpha_{n} Z_{n}^{k}}\right) \mathbf{1}_{\left\{Z_{1}^{k}=0=\cdots=Z_{k-1}^{k}=0, Z_{k}^{k}>0\right\}}\left(Z_{k}^{k}\right)^{-1}\right] \\
= & -\alpha_{n} c_{n} \\
& -\sum_{k=1}^{n} \theta_{k} \int_{\mathbb{R}_{+}^{n}}\left[e^{-\sum_{i=1}^{n-1} \alpha_{i} y_{i}}-e^{-\sum_{i=1}^{n} \alpha_{i} y_{i}}\right] \mathbf{1}_{\left\{y_{1}=\cdots=y_{k-1}=0, y_{k}>0\right\}} y_{k}^{-1} \mathscr{L}\left(Z^{k}\right)(d y) \\
= & -\alpha_{n} c_{n}+\int_{\mathbb{R}_{+}^{n}}\left(1-e^{-\sum_{i=1}^{n-1} \alpha_{i} y_{i}}\right) v(d y)-\int_{\mathbb{R}_{+}^{n}}\left(1-e^{-\sum_{i=1}^{n} \alpha_{i} y_{i}}\right) v(d y) .
\end{aligned}
$$

Substituting the above into (6.13) completes the proof.

Proof of Proposition 4.7. The form of the drift follows from Lemma 4.6. Let $I=\left\{t_{1}, \ldots, t_{n}\right\} \subset T$. We have

$$
\int_{\mathbb{R}_{+}^{T}}\left(1-e^{-\sum_{i=1}^{n} \alpha_{i} y\left(t_{i}\right)}\right) v(d y)=\sum_{k \geq 1} \int_{\mathbb{R}_{+}^{T}}\left(1-e^{-\sum_{i=1}^{n} \alpha_{i} y\left(t_{i}\right)}\right) v_{k}(d y)
$$

$$
=\sum_{k \geq 1} \sum_{j=1}^{n} \int_{\mathbb{R}_{+}^{T}}\left(1-e^{-\sum_{i=1}^{n} \alpha_{i} y\left(t_{i}\right)}\right) \mathbf{1}_{\left\{y\left(t_{1}\right)=\cdots=y\left(t_{j-1}\right)=0, y\left(t_{j}\right)>0\right\}} v_{k}(d y),
$$

and by (6.12), for every $j, k$,

$$
\begin{aligned}
\int_{\mathbb{R}_{+}^{T}}(1 & \left.-e^{-\sum_{i=1}^{n} \alpha_{i} y\left(t_{i}\right)}\right) \mathbf{1}_{\left\{y\left(t_{1}\right)=\cdots=y\left(t_{j-1}\right)=0, y\left(t_{j}\right)>0\right\}} v_{k}(d y) \\
= & \theta\left(s_{k}\right) \mathbb{E}\left[\left(1-e^{-\sum_{i=1}^{n} \alpha_{i} Z_{t_{i}}^{s_{k}}}\right)\right. \\
& \left.\times \mathbf{1}_{\left\{Z_{s_{1}}^{s_{k}}=\cdots=Z_{s_{k-1}}^{s_{k}}=0, Z_{s_{k}}^{s_{k}}>0, Z_{t_{1}}^{s_{k}}=\cdots=Z_{t_{j-1}}^{s_{k}}=0, Z_{t_{j}}^{s_{k}}>0\right\}}\left(Z_{s_{k}}^{s_{k}}\right)^{-1}\right] \\
= & \theta\left(t_{j}\right) \mathbb{E}\left[\left(1-e^{\left.-\sum_{i=1}^{n} \alpha_{i} Z_{t_{i}}^{t_{j}}\right)}\right.\right. \\
& \left.\times \mathbf{1}_{\left\{Z_{s_{1}}^{t_{j}}=\cdots=Z_{s_{k-1}}^{t_{j}}=0, Z_{s_{k}}^{t_{j}}>0, Z_{t_{1}}^{t_{j}}=\cdots=Z_{t_{j-1}}^{t_{j}}=0, Z_{t_{j}}^{t_{j}}>0\right\}}\left(Z_{t_{j}}^{t_{j}}\right)^{-1}\right] .
\end{aligned}
$$


Substituting this into (6.15) and summing over $k$ gives

$$
\begin{aligned}
& \int_{\mathbb{R}_{+}^{T}}(\left.1-e^{-\sum_{i=1}^{n} \alpha_{i} y\left(t_{i}\right)}\right) v(d y) \\
& \quad=\sum_{j=1}^{n} \theta\left(t_{j}\right) \mathbb{E}\left(1-e^{-\sum_{i=1}^{n} \alpha_{i} Z_{t_{i}}^{t_{j}}}\right) \mathbf{1}_{\left\{Z_{T_{0}}^{t_{j}} \neq 0\right\}} \mathbf{1}_{\left\{Z_{t_{1}}^{t_{j}}=0, \ldots, Z_{t_{j-1}}^{t_{j}}=0, Z_{t_{j}}^{t_{j}}>0\right\}}\left(Z_{t_{j}}^{t_{j}}\right)^{-1} .
\end{aligned}
$$

In view of Lemma 4.6, we now need to show that $\left\{Z_{T_{0}}^{t_{j}} \neq 0\right\}=\left\{Z_{t_{j}}^{t_{j}}>0\right\}$ a.s. To this aim, we first notice that $\left\{Z_{T_{0}}^{t_{j}} \neq 0\right\} \subset\left\{Z_{t_{j}}^{t_{j}}>0\right\}$ a.s. by (6.11). Then, choose $\left(s_{k_{n}}\right)_{n \geq 1} \subset T_{0}$ such that $Y_{s_{n}} \stackrel{P}{\rightarrow} Y_{t_{j}}$. Using (4.18) for $F(y)=\exp \left(-\alpha\left(y\left(s_{k_{n}}\right)-\right.\right.$ $\left.\left.y\left(t_{j}\right)\right)\right), \alpha>0$ we get $Z_{s_{n}}^{t_{j}} \stackrel{P}{\rightarrow} Z_{t_{j}}^{t_{j}}$ as $n \rightarrow \infty$. Hence,

$$
\mathbb{P}\left(Z_{t_{j}}^{t_{j}}>0\right) \leq \liminf _{n \rightarrow \infty} \mathbb{P}\left(Z_{s_{k_{n}}}^{t_{j}}>0\right) \leq \mathbb{P}\left(Z_{T_{0}}^{t_{j}}>0\right) \leq \mathbb{P}\left(Z_{t_{j}}^{t_{j}}>0\right) .
$$

Since $v$ determines all finite dimensional distributions of $Y$ and clearly $v\left\{y: y_{T_{0}}=\right.$ $0\}=0, v$ is the Lévy measure of $Y$.

To complete the proof, consider a nonnegative infinitely divisible process $\left(Y_{t}\right)_{t \in T}$ with finite mean $\theta(t)=\mathbb{E} Y_{t}$. Let $c$ and $v$ be the drift and Lévy measure of $Y$, respectively. Similar to the proof of Lemma 4.6(i), we check that (4.20) satisfies (4.18). The proof is complete.

Proof of Proposition 4.13. Let $X$ be determined by (4.21), where we take $\llbracket v \rrbracket=v \mathbf{1}_{[-1,1]}(v)$ for concreteness. Given $h>0$, let $F: \mathbb{R}^{[0, h]} \mapsto \mathbb{R}$ be defined by $F(x)=f(x(h))$. By (4.22), we have

$$
h^{-1} \mathbb{E}\left[f\left(X_{h}\right) ; W_{h}>0\right]=\int_{\mathbb{R}} \mathbb{E}\left[f\left(X_{h}+v\right) ;\left(W_{h}+q_{1}(v)\right)^{-1}\right] q_{1}(v) \rho(d v) .
$$

Choose $q_{1}=m^{-1} \mathbf{1}_{\{|v|>\delta\}}$, where $\delta \in(0,1)$ is fixed and $m:=\rho\{|v|>\delta\}>0$. Then we have

$$
\begin{aligned}
h^{-1} \mathbb{E} & {\left[f\left(X_{h}\right)\right] } \\
& =h^{-1} \mathbb{E}\left[f\left(X_{h}\right) ; W_{h}=0\right]+\int_{|v|>\delta} \mathbb{E}\left[f\left(X_{h}+v\right) ;\left(m W_{h}+1\right)^{-1}\right] \rho(d v) .
\end{aligned}
$$

Hence

$$
\begin{aligned}
\mid h^{-1} \mathbb{E}[ & \left.f\left(X_{h}\right)\right]-\int_{\mathbb{R}} f(v) \rho(d v) \mid \\
\leq & h^{-1}\left|\mathbb{E}\left[f\left(X_{h}\right) ; W_{h}=0\right]\right|+\int_{|v| \leq \delta}|f(v)| \rho(d v) \\
& +\int_{\{|v|>\delta\}} \mathbb{E}\left|f\left(X_{h}+v\right)\left(m W_{h}+1\right)^{-1}-f(v)\right| \rho(d v) \\
:= & K_{1}(h, \delta)+K_{2}(\delta)+K_{3}(h, \delta) .
\end{aligned}
$$


Notice that if $W_{h}=0$, then $X_{h}=X_{h}^{\delta}$, where $X_{h}^{\delta}=X_{h}-\sum_{s \leq h} \Delta X_{s} \mathbf{1}\left(\left|\Delta X_{s}\right|>\delta\right)$. $X_{h}^{\delta}$ is a Lévy process with variance $\sigma^{2}$ of its Brownian motion component $\left\{G_{t}\right\}$, Lévy measure $\rho=\rho_{\mid\{|x| \leq \delta\}}$ and the shift $c^{\delta}=c-\int_{\delta<|x| \leq 1} x \rho(d x)$, so that $\mathbb{E} X_{h}^{\delta}=$ $c^{\delta} h$. Put $Y_{h}^{\delta}=X_{h}^{\delta}-G_{h}$. By the assumption, $|f(x)| \leq x^{2} k(x)$, where $0 \leq k(x) \leq C$ is a bounded function with $\lim _{x \rightarrow 0} k(x)=0$, or only a bounded function when $\sigma^{2}=0$. We get

$$
\begin{aligned}
K_{1}(h, \delta) & \leq h^{-1} \mathbb{E}\left(\left|X_{h}^{\delta}\right|^{2} k\left(X_{h}^{\delta}\right)\right) \leq 2 h^{-1} \mathbb{E}\left(\left|G_{h}\right|^{2} k\left(X_{h}^{\delta}\right)\right)+2 C h^{-1} \mathbb{E}\left(\left|Y_{h}^{\delta}\right|^{2}\right) \\
& \leq 2 h^{-1}\left(\mathbb{E}\left|G_{h}\right|^{4}\right)^{1 / 2}\left(\mathbb{E}\left|k\left(X_{h}^{\delta}\right)\right|^{2}\right)^{1 / 2}+2 C h^{-1}\left(\operatorname{Var}\left(Y_{h}^{\delta}\right)+\left|\mathbb{E} Y_{h}^{\delta}\right|^{2}\right) \\
& =2 \sqrt{3} \sigma^{2}\left(\mathbb{E}\left|k\left(X_{h}^{\delta}\right)\right|^{2}\right)^{1 / 2}+2 C \operatorname{Var}\left(Y_{1}^{\delta}\right)+2 C\left|c^{\delta}\right|^{2} h \\
& \stackrel{h \rightarrow 0}{\longrightarrow} 2 C \int_{\{|v| \leq \delta\}} v^{2} \rho(d v) .
\end{aligned}
$$

We see that when $\sigma^{2}=0$ we only need $k$ to be bounded. Then $K_{2}(\delta) \leq$ $C \int_{\{|v| \leq \delta\}} v^{2} \rho(d v)$ and $K_{3}(h, \delta) \rightarrow 0$ as $h \rightarrow 0$ because $X_{h}, W_{h} \rightarrow 0$ a.s. and continuous on a set of full $\rho$-measure. Combining the above estimates, we get

$$
\limsup _{h \rightarrow 0}\left|h^{-1} \mathbb{E}\left[f\left(X_{h}\right)\right]-\int_{\mathbb{R}} f(v) \rho(d v)\right| \leq 3 C \int_{\{|v| \leq \delta\}} v^{2} \rho(d v) .
$$

Letting $\delta \rightarrow 0$ gives (4.23).

Finally, notice that if $X$ is a subordinator, then in the term $K_{3}(h, \delta), X_{h}+v$ approaches $v$ from the right. Thus, if $f$ is right-continuous on a set of full $\rho$ measure we get also $\lim _{h \rightarrow 0} K_{1}(h, \delta)=0$. This completes the proof.

Proof OF TheOREM 5.6. Recall that $Y$ has the generating triplet $(0, v, b)$ and $V$ is a representation of $v$ on a $\sigma$-finite measure space $(S, \mathscr{S}, n) .\left(\xi_{j}\right)_{j \in \mathbb{N}}$ is an i.i.d. sequence of random elements in $S$ with the common distribution $g(s) n(d s)$, where $g>0 n$-a.e. Finally, $\left(\Gamma_{j}\right)_{j \in \mathbb{N}}$ is a sequence of partial sums of i.i.d. standard exponential random variables independent of $\left(\xi_{j}\right)_{j \in \mathbb{N}}$.

We will show the isomorphism identities by a reduction to the stochastic integral case. To this aim, consider $\bar{S}=\left\{(s, r) \in S \times \mathbb{R}_{+}: 0 \leq r \leq g(s)^{-1}\right\}$ with the measure $\bar{n}(d s, d r):=g(s) n(d s) d r$ and let $\bar{V}_{t}(s, r):=V_{t}(s)$. Then $\bar{V}$ is a representation a representation of $\nu$. Indeed, for any $A \in \mathbb{R}^{T}$

$$
\bar{n} \circ \bar{V}^{-1}(A)=\int_{S} \int_{0}^{g(s)^{-1}} \mathbf{1}_{A}(V(s)) g(s) d r n(d s)=n \circ V^{-1}(A)=v(A) .
$$

A Poisson random measure $N:=\sum_{j=1}^{\infty} \delta_{\left(\xi_{j}, \Gamma_{j}\right)}$ on $S \times \mathbb{R}_{+}$has the intensity measure $g(s) n(d s) d r$, so the restriction of $N$ to $\bar{S}$ has the intensity $\bar{n}$. We have

$$
Y_{t}=\int_{\bar{S}} V_{t}(s)\left[\bar{N}(d s, d r)-\chi\left(V_{t}(s)\right) \bar{n}(d s, d r)\right]+b(t) \quad \text { a.s. }
$$


Therefore, using (4.8) of Theorem 4.4 we get

$$
\begin{aligned}
\mathbb{E}\left[F\left(\left(V_{t}\left(\xi_{0}\right)+Y_{t}\right)_{t \in T}\right)\right] & =\mathbb{E} \int_{S}\left[F\left(\left(V_{t}(s)+Y_{t}\right)_{t \in T}\right)\right] q(s) n(d s) \\
& =\mathbb{E} \int_{S} \int_{0}^{g(s)^{-1}}\left[F\left(\left(V_{t}(s)+Y_{t}\right)_{t \in T}\right)\right] g(s) q(s) d r n(d s) \\
& =\mathbb{E} \int_{\bar{S}}\left[F\left(\left(V_{t}(s)+Y_{t}\right)_{t \in T}\right)\right] q(s) \mathbf{1}\left\{r \leq g(s)^{-1}\right\} \bar{n}(d s, d r) \\
& =\mathbb{E}\left[F\left(\left(Y_{t}\right)_{t \in T}\right) ; \bar{q}(\bar{N})\right],
\end{aligned}
$$

where $\bar{q}(s, r)=q(s) \mathbf{1}\left\{r \leq g(s)^{-1}\right\}$ and

$$
\bar{N}(\bar{q})=\int_{\bar{S}} q(s) \mathbf{1}\left\{g(s) \leq r^{-1}\right\} \bar{N}(d s, d r)=\sum_{j=1}^{\infty} q\left(\xi_{j}\right) \mathbf{1}\left\{g\left(\xi_{j}\right) \leq \Gamma_{j}^{-1}\right\}=Q .
$$

This proves (5.6).

Conversely, using (4.9) of Theorem 4.4 we get

$$
\begin{aligned}
\mathbb{E}[F( & \left.\left.\left(Y_{t}\right)_{t \in T}\right) ; Q>0\right] \\
& =\mathbb{E}\left[F\left(\left(Y_{t}\right)_{t \in T}\right) ; \bar{N}(\bar{q})>0\right] \\
& =\mathbb{E} \int_{\bar{S}}\left[F\left(\left(V_{t}(s)+Y_{t}\right)_{t \in T}\right) ;(\bar{N}(\bar{q})+\bar{q}(s, r))^{-1}\right] \bar{q}(s, r) \bar{n}(d s, d r) \\
& =\mathbb{E} \int_{S} \int_{0}^{g(s)^{-1}}\left[F\left(\left(V_{t}(s)+Y_{t}\right)_{t \in T}\right) ;(Q+q(s))^{-1}\right] q(s) \operatorname{drg}(s) n(d s) \\
& =\mathbb{E} \int_{S}\left[F\left(\left(V_{t}(s)+Y_{t}\right)_{t \in T}\right) ;(Q+q(s))^{-1}\right] q(s) n(d s) .
\end{aligned}
$$

The last statement of the theorem follows from the corresponding statement in Theorem 4.4.

Acknowledgments. The author is grateful to Nathalie Eisenbaum, Haya Kaspi, Michael B. Marcus and Jay Rosen for the stimulating discussions on various topics related to permanental processes. He is also grateful to the anonymous referee for useful remarks and calling our attention to [7], Lemma 3.1, where an abstract form of Dynkin's isomorphism was introduced and considered.

\section{REFERENCES}

[1] Applebaum, D. and Riedle, M. (2010). Cylindrical Lévy processes in Banach spaces. Proc. Lond. Math. Soc. (3) 101 697-726. MR2734958

[2] Barndorff-Nielsen, O. E., SAuri, O. and Szozda, B. (2017). Selfdecomposable fields. J. Theoret. Probab. 30 233-267. MR3615088 
[3] BASSE-O'Connor, A. and Rosiński, J. (2013). On the uniform convergence of random series in Skorohod space and representations of càdlàg infinitely divisible processes. Ann. Probab. 41 4317-4341. MR3161476

[4] Cambanis, S., Nolan, J. P. and Rosiński, J. (1990). On the oscillation of infinitely divisible and some other processes. Stochastic Process. Appl. 35 87-97. MR1062585

[5] Dynkin, E. B. (1984). Gaussian and non-Gaussian random fields associated with Markov processes. J. Funct. Anal. 55 344-376.

[6] Eisenbaum, N. (2003). On the infinite divisibility of squared Gaussian processes. Probab. Theory Related Fields 125 381-392.

[7] Eisenbaum, N. (2008). A Cox process involved in the Bose-Einstein condensation. Ann. Henri Poincaré 9 1123-1140.

[8] Eisenbaum, N. and KasPi, H. (2006). A characterization of the infinitely divisible squared Gaussian processes. Ann. Probab. 34 728-742. MR2223956

[9] Eisenbaum, N. and KasPi, H. (2009). On permanental processes. Stochastic Process. Appl. 119 1401-1764.

[10] KabluchKo, Z. and Stoev, S. (2016). Stochastic integral representations and classification of sum- and max-infinitely divisible processes. Bernoulli 22 107-142. MR3449778

[11] Kallenberg, O. (2002). Foundations of Modern Probability, 2nd ed. Springer, New York. MR1876169

[12] LEE, P. M. (1967). Infinitely divisible stochastic processes. Z. Wahrsch. Verw. Gebiete $7147-$ 160.

[13] Mansuy, R. and Yor, M. (2008). Aspects of Brownian Motion. Springer, Berlin.

[14] Marcus, M. B. and Rosen, J. (1992). Sample path properties of the local times of strongly symmetric Markov processes via Gaussian processes. Ann. Probab. 20 1603-1684. MR1188037

[15] Marcus, M. B. and Rosen, J. (2006). Markov Processes, Gaussian Processes, and Local Times. Cambridge Studies in Advanced Mathematics 100. Cambridge Univ. Press, Cambridge. MR2250510

[16] Maruyama, G. (1970). Infinitely divisible processes. Theory Probab. Appl. 15 1-22. MR0285046

[17] Pitman, J. W. and Yor, M. (1982). A decomposition of Bessel bridges. Z. Wahrsch. Verw. Gebiete 59 425-457.

[18] RAJPut, B. S. and Rosiński, J. (1989). Spectral representations of infinitely divisible processes. Probab. Theory Related Fields 82 451-487.

[19] Revuz, D. and Yor, M. (1999). Continuous Martingales and Brownian Motion, 3rd ed. Grundlehren der Mathematischen Wissenschaften 293. Springer, Berlin.

[20] RosińsKI, J. (1990). On series representations of infinitely divisible random vectors. Ann. Probab. 18 405-430. MR1043955

[21] RosińSKI, J. (1990). An application of series representations for zero-one laws for infinitely divisible random vectors. In Probability in Banach Spaces 7. Progr. Probab. 21 189-199. Birkhäuser, Boston, MA. MR1105559

[22] Rosiński, J. (2001). Series representations of Lévy processes from the perspective of point processes. In Lévy Processes 401-415. Birkhäuser, Boston, MA.

[23] RosińsKI, J. (2007). Lévy and related jump-type infinitely divisible processes. Lecture Notes, Cornell Univ., Ithaca, NY.

[24] SAto, K. (1999). Lévy Processes and Infinitely Divisible Distributions. Cambridge Studies in Advanced Mathematics 68. Cambridge Univ. Press, Cambridge.

[25] Shiga, T. and Watanabe, S. (1973). Bessel diffusions as a one-parameter family of diffusion processes. Z. Wahrsch. Verw. Gebiete 27 37-46. MR0368192

[26] Talagrand, M. (2014). Upper and Lower Bounds for Stochastic Processes. Modern Surveys in Mathematics 60. Springer, Heidelberg. MR3184689 
[27] Vere-Jones, D. (1967). The infinite divisibility of a bivariate gamma distributions. Sankhya Ser. A 29 421-422. MR0226704

DEPARTMENT OF MATHEMATICS UNIVERSITY OF TENNESSEE KNOXVILle, TennesseE 37996 USA

E-MAIL: rosinski@utk.edu 